\title{
Ballistic transport and electronic structure
}

\author{
Kees M. Schep* \\ Faculty of Applied Physics and Delft Institute of Microelectronics and Submicrontechnology, Delft University of Technology, \\ Lorentzweg 1, 2628 CJ Delft, The Netherlands \\ Paul J. Kelly ${ }^{\dagger}$ \\ Philips Research Laboratories, Prof. Holstlaan 4, 5656 AA Eindhoven, The Netherlands \\ Gerrit E. W. Bauer \\ Faculty of Applied Physics and Delft Institute of Microelectronics and Submicrontechnology, Delft University of Technology, \\ Lorentzweg 1, 2628 CJ Delft, The Netherlands
}

(Received 10 September 1997)

\begin{abstract}
The role of the electronic structure in determining the transport properties of ballistic point contacts is studied. The conductance in the ballistic regime is related to simple geometrical projections of the Fermi surface. The essential physics is first clarified for simple models. For real materials the band structure is taken into account using parameter-free local-spin-density approximation calculations. In magnetic metallic multilayers the electronic structure gives rise to a large difference in conductance between the parallel and antiparallel configurations. For $\mathrm{Co} / \mathrm{Cu}$ and $\mathrm{Fe} / \mathrm{Cr}$ multilayers the dependence of the conductances on the layer thicknesses and the crystal orientations is investigated for the geometries with the current perpendicular, parallel, and at an angle to the interface planes. In spite of the absence of spin-dependent scattering at defects, the ballistic giant magnetoresistance effects in the perpendicular geometry can be as large as $120 \%$ and $230 \%$ in $\mathrm{Co} / \mathrm{Cu}$ and $\mathrm{Fe} / \mathrm{Cr}$ multilayers, respectively. [S0163-1829(98)04612-8]
\end{abstract}

\section{INTRODUCTION}

Most experimental studies of electrical transport are in the diffusive transport regime, in which the sample dimensions are much larger than the mean free path. In this regime the conductivity is determined both by the electronic structure of the material and by the scattering at defects. This can be illustrated within the free-electron model with two commonly used expressions for the Drude conductivity,

$$
\sigma_{\text {Drude }}=e^{2}\left\{\frac{n}{m}\right\} \tau=\frac{2 e^{2}}{h}\left\{\frac{k_{F}^{2}}{3 \pi}\right\} l .
$$

The diffusive conductivity depends both on electronic structure parameters (in curly brackets) such as the Fermi wave vector $k_{F}$ or the ratio between the density $n$ and the mass $m$ of the electrons, and on scattering parameters such as the mean free path 1 or the relaxation time $\tau$. This division into an electronic structure part and a scattering part is not unique since the electronic structure also plays a role in determining the scattering properties. The relaxation time, for example, depends on the density of states at the Fermi energy. The factorization of Eq. (1) into electronic structure and scattering contributions is an artifact of the free-electron model. For general band structures and scattering mechanisms the two aspects are strongly entwined and a simple factorization does not exist. This complicates the evaluation of the effect that the electronic structure has on the transport properties.

In the ballistic transport regime the sample dimensions are much smaller than the mean free path. In this regime the conductance does not depend on the scattering properties but only on the band structure and on the device geometry. It was pointed out by Sharvin ${ }^{1}$ that electrical transport in ballistic samples is a sensitive tool to study Fermi surface properties, as has been demonstrated by electron focusing experiments. $^{2,3}$ In studies of electrical transport through single ballistic point contacts attention has often been focussed on the nonlinear phenomena in the current-voltage characteristics. The observed deviations from Ohm's law can be used for a spectral analysis of the interaction mechanisms of the conduction electrons with elementary excitations in metals. ${ }^{4-6}$ The conductance in the linear response regime is just a constant that appears to be not very interesting. Even in relatively well defined microfabricated point contacts ${ }^{7,8}$ the cross-sectional area of the contact is not accurately known. This prevents the experimental determination of the conductance per unit area, which is a material specific parameter that contains information on the electronic structure. In fact, usually a theoretical estimate for this parameter is used to determine the area of the cross section from the measured conductance.

Recently, we identified a situation in which the measurement of the conductance of a single ballistic point contact gives rise to interesting new effects in the linear response regime. ${ }^{9}$ For magnetic metallic multilayers consisting of alternating magnetic and nonmagnetic layers and the current flowing perpendicular to the interface planes we predicted that the conductance in the ballistic regime should increase by more than a factor of 2 when the relative orientation of the magnetizations of adjacent magnetic layers is changed from antiparallel to parallel. This change in conductance arises from the difference in electronic structure between the antiparallel and the parallel configuration. The alignment of the magnetization can be realized experimentally by apply- 
ing an external magnetic field to antiferromagnetically coupled magnetic multilayers. The large change in resistance induced by applying a magnetic field to such systems is referred to as the giant magnetoresistance (GMR) effect. Even if the cross-sectional area of the point contact is not known, the relative resistance change can still be measured accurately since the experiment can be done using a single contact.

Experimentally the GMR effect ${ }^{10-12}$ has up to now only been observed in the diffusive transport regime. Some initial experiments ${ }^{13-15}$ on very small samples have been performed but thus far ballistic transport in magnetic multilayers has not been realized. The GMR effect has been the subject of intense investigation mainly because of the advantages it promises for magnetic recording and sensing. Most measurements are made in the geometry with the current flowing parallel to the interface planes, the so-called current-in-plane (CIP) geometry. The experimentally more challenging current-perpendicular-to-plane (CPP) geometry ${ }^{16-18}$ results in an enhanced GMR and should also help to clarify the origin of the effect because of the higher symmetry and the clearer role of the interfaces as compared to the CIP geometry. In addition to the CIP and CPP geometries the conductance can also be measured in the so-called CAP geometry ${ }^{19}$ in which the current flows at an angle to the interface planes.

There is consensus about the conditions under which the GMR effect occurs, namely, when the magnetizations of neighboring ferromagnetic layers, which are initially oriented antiparallel or at random, are aligned by applying an external magnetic field. A satisfactory microscopic explanation of the physical processes causing the GMR is still lacking, however. Most theories that attempt to explain the GMR by spin-dependent scattering at defects, either in the bulk or at the interfaces, do not treat the underlying electronic structure realistically. Instead, transport is assumed to be mediated by the $s$ electrons, which are described by a parabolic band with some appropriate effective mass ${ }^{20-30}$ or by a nondegenerate tight-binding band. ${ }^{31-33}$ The magnetism, which is associated with the tightly bound $d$ electrons, is introduced in terms of phenomenological scattering or tight-binding parameters. Studies of transport in the diffusive regime that do take into account the complicated band structure of the multilayer ${ }^{34-39}$ suffer from strong intertwining of electronic structure and scattering effects that is inherent to the diffusive regime and that complicates the interpretation of the results. A clear distinction between band structure and scattering contributions can only be obtained at the cost of making approximations in the transport theory, such as the stateindependent relaxation-time approximation. ${ }^{34,37}$ The validity of such approximations is, however, not clear. Furthermore, the choice of a realistic model for the disorder, either microscopic or phenomenological, is far from trivial.

In this paper we calculate the conductance of a ballistic point contact in the linear response regime with emphasis on its dependence on the electronic structure. In addition to model calculations that clarify the basic physics we carry out first-principles electronic structure calculations based on the local-spin-density approximation. The parameter-free results for the ballistic conductance can in principle be compared directly with experiment. For magnetic multilayers we find that a perfectly periodic structure without any defects sup- ports a giant MR. This is in contrast with the common belief that GMR is mainly due to spin-dependent defect scattering. For $\mathrm{Co} / \mathrm{Cu}$ multilayers this surprising result can be ascribed almost entirely to the fact that we realistically take into account the hybridization between the free-electron-like $s$ electrons and the heavier $d$ electrons (see Sec. V C); this hybridization is neglected in the simple theories. Before trying to understand the GMR in dirty multilayers it would be very desirable to have some insight into the transport properties of ideal multilayers. In this paper an attempt is made to provide such insight. Besides being of interest for future experiments in the ballistic regime we believe that our calculations are also relevant for the interpretation of present measurements in the diffusive regime.

The method that we use to study ballistic transport in magnetic multilayers can be applied straightforwardly to other materials. Gómez Abal, Llois, and Weissmann ${ }^{40}$ calculated the ballistic magnetoresistance in the FeRh intermetallic compound. The magnetic moments on the $\mathrm{Fe}$ sites in FeRh are coupled antiferromagnetically but can be aligned by applying an external magnetic field. The corresponding magnetoresistance calculated in Ref. 40 is as large as $400 \%$. Experimentally, ${ }^{41}$ the dependence of the resistance on the external magnetic field is highly nonmonotonic. A theoretical description of this dependence is still lacking but will require the self-consistent calculation of the (probably noncollinear) magnetic structure of $\mathrm{FeRh}$ as a function of the external magnetic field. The changes in resistance that accompany the metamagnetic phase transitions in both magnetic multilayers and intermetallic compounds are induced by changes in electronic structure. The same mechanism accounts for the magnetoresistances that are calculated in the diffusive regime using a spin- and state-independent relaxation-time approximation for multilayers, ${ }^{34,37}$ the $\mathrm{FeRh}$ compound, ${ }^{40}$ and uranium compounds. ${ }^{42,43}$ This mechanism was invoked earlier to explain resistance anomalies around the magnetic ordering temperatures in rare-earth metals. ${ }^{44,45}$ Weissmann et al. ${ }^{46}$ considered the ballistic transport in $\mathrm{Co} / \mathrm{Ni}$ multilayers in which all layers are magnetic and strongly ferromagnetically coupled. This system does not go through a metamagnetic phase transition and therefore does not exhibit a giant magnetoresistance effect. The $\mathrm{Co} / \mathrm{Ni}$ multilayers are of interest because of the oscillatory behavior of the resistance as a function of the layer thicknesses. ${ }^{47}$

Mathon $^{48}$ has calculated ballistic transport through $\mathrm{Co} / \mathrm{Cu}$ and $\mathrm{Fe} / \mathrm{Cr}$ multilayers of finite thickness sandwiched between infinite leads of $\mathrm{Cu}$ and $\mathrm{Cr}$, respectively. The values he calculates for the MR are similar but not identical to the ones we obtained in Ref. 9. This discrepancy is partly due to small differences in the electronic structure ${ }^{48}$ but, more importantly, also due to the difference between the "finite" geometry considered by Mathon and the "infinite" geometry considered by us; these two geometries are depicted schematically in Figs. 2(a) and 2(b), respectively, of Ref. 49 (not shown). Similarly, different experimental geometries (such as "finite," "infinite," or as in Ref. 14 "semi-infinite") should be distinguished. Direct comparison between theory and experiment is possible only for the same geometry. The similarity between our results and those of Mathon ${ }^{48}$ indicates, however, that the effect of the geometry on the results obtained so far is only minor. Besides the ballistic regime, 
Mathon also considered transport through multilayers with fluctuating layer thicknesses. ${ }^{48}$

The organization of this paper is as follows. In Sec. II derivations are presented of the expression for the ballistic conductance that is valid for a general electronic structure. This expression is evaluated in Sec. III for free-electron, tight-binding, and Kronig-Penney models and in Sec. IV for realistic bulk materials using first-principles band-structure calculations. The ballistic transport and magnetoresistance in $\mathrm{Co} / \mathrm{Cu}$ and $\mathrm{Fe} / \mathrm{Cr}$ multilayers are discussed in Secs. V and VI, respectively. Our results are summarized in Sec. VII. Short accounts of part of this work were given in Ref. 9 and a number of conference proceedings. ${ }^{49-52}$

\section{BALLISTIC TRANSPORT}

In this section the general expression for the ballistic conductance is derived. Consider two semi-infinite electrodes separated by an insulating barrier and only connected via a small opening in the barrier. This device is referred to as a classical ballistic point contact when the diameter of the opening is much smaller than the mean free path and much larger than the electron wavelength. ${ }^{53}$ The resistance of such a point contact is determined by the ballistic motion of the electrons through the opening. ${ }^{1}$ Even though the electrons passing through the constriction are not scattered out of their Bloch states, the conductance of the point contact is finite due to its finite cross section $A$. A sufficiently small opening is a small perturbation to the unconnected electrodes and, in lowest order, does not disturb the equilibrium distribution functions of the electrons in the two electrodes. The net current is given by the difference in the number of electrons incident upon the opening from each side per unit time. Thus for a small voltage difference $V$ between the electrodes (and at low temperatures) the current $I$ in the transport direction $\hat{n}$ is $^{6}$

$$
I=\operatorname{AeVe} \frac{1}{2} \sum_{\nu \sigma} \int \frac{d \vec{q}}{8 \pi^{3}}\left|\hat{n} \cdot \vec{v}_{\nu \sigma}(\vec{q})\right| \delta\left(\varepsilon_{\nu \sigma}(\vec{q})-E_{F}\right)
$$

where $\vec{v}_{\nu \sigma}(\vec{q})$ and $\varepsilon_{\nu \sigma}(\vec{q})$ are the velocity and the energy, respectively, for a state with Bloch vector $\vec{q}$, band index $\nu$, and spin index $\sigma$. The velocity of a Bloch state can be obtained from the band structure using

$$
\vec{v}_{\nu \sigma}(\vec{q})=\frac{1}{\hbar} \nabla_{\vec{q}} \varepsilon_{\nu \sigma}(\vec{q})
$$

The factor $1 / 2$ in Eq. (2) appears because only electrons moving towards the opening contribute to the current. The integration over $\vec{q}$ can be replaced by an integration over the corresponding sheet of the Fermi surface FS $(\nu \sigma)$. The ballistic or Sharvin conductance $G(\hat{n})=I / V$ can then be written as $^{54}$

$$
G(\hat{n})=\frac{e^{2}}{h} \frac{A}{4 \pi^{2}} \frac{1}{2} \sum_{\nu \sigma} \int_{\mathrm{FS}(\nu \sigma)} \frac{d S}{\left|\overrightarrow{v_{\nu \sigma}}(\vec{q})\right|}\left|\hat{n} \cdot \vec{v}_{\nu \sigma}(\vec{q})\right|
$$

Since $\vec{v}_{\nu \sigma}(\vec{q})$ at $E_{F}$ is always normal to the Fermi surface, $G(\hat{n})$ can be expressed in terms of the projections $S_{\nu \sigma}(\hat{n})$ of $\mathrm{FS}(\nu \sigma)$ in the direction $\hat{n}$ :

$$
G(\hat{n})=\frac{e^{2}}{h} \frac{A}{4 \pi^{2}} \frac{1}{2} \sum_{\nu \sigma} S_{\nu \sigma}(\hat{n}) .
$$

The above derivation of the ballistic conductance is analogous to the calculation of molecular effusion of a dilute gas through a small hole in response to a pressure gradient. The amount of gas that flows through the aperture per unit time is in that case directly related to the equilibrium velocity distribution function of the molecules. Experiments in the regime where the mean free path of the gas is much larger than the dimensions of the hole were undertaken in 1908 by Knudse ${ }^{55}$ to test the predictions of the kinetic theory of gases. The ballistic regime of electrical transport is therefore sometimes referred to as the Knudsen regime and the Sharvin conductance as the Knudsen conductance. ${ }^{54}$

The expression for the ballistic conductance can alternatively be derived from the Landauer-Büttiker formalism. ${ }^{56}$ For a general band structure the Landauer conductance formula can be written in terms of incoming and outgoing Bloch states that are labeled by the component $\vec{q}_{\|}$of the Bloch vector parallel to the insulating plane (i.e., perpendicular to $\hat{n}$ ) and by the indices $\nu$ and $\sigma$ :

$$
G(\hat{n})=\frac{e^{2}}{h} \sum_{\vec{q}_{\|} \nu \sigma, \vec{q}_{\|}^{\prime} \nu^{\prime} \sigma^{\prime}}\left|t_{\vec{q}_{\|} \nu \sigma, \vec{q}_{\|}^{\prime} \nu^{\prime} \sigma^{\prime}}\right|^{2} .
$$

The calculation of the transmission probabilities $\left|t_{q_{\|} \nu \sigma, \vec{q}_{\|}^{\prime} \nu^{\prime} \sigma^{\prime}}\right|^{2}$ from the incident mode $\vec{q}_{\|}^{\prime} \nu^{\prime} \sigma^{\prime}$ to the transmitted mode $\vec{q}_{\|} \nu \sigma$ is in general difficult. In the ballistic regime, however, this calculation is trivial because the modes are not scattered at all and the transmission probability matrix is simply the unit matrix. The conductance then becomes

$$
G(\hat{n})=\frac{e^{2}}{h} \sum_{\sigma} N_{\sigma}(\hat{n}),
$$

where $N(\hat{n})$ is the number of conducting channels for transport in direction $\hat{n}$. The number of channels can be counted by noting that the density of transverse modes equals $A / 4 \pi^{2}$ and that only the propagating modes moving towards the contact should be taken into account. This gives Eq. (5) again and thus the two derivations yield the same result.

The above discussion is limited to the three-dimensional case. For electrical transport in two-dimensional electron gases in semiconductor nanostructures ${ }^{57,58}$ the twodimensional case is relevant. In two dimensions $A$ is the width of the constriction and the density of transverse modes equals $A / 2 \pi$. The right-hand sides of Eqs. (2), (4), and (5) should therefore be multiplied by a factor $2 \pi$ in the twodimensional case.

We briefly compare the formalism for the ballistic regime with an approach used by others to study transport in the diffusive regime where scattering at defects should be taken into account. Defect scattering in magnetic multilayers has not yet been calculated from first principles and approxima- 
tions have been employed instead. In the relaxation-time approximation to the Boltzmann equation the diagonal component of the conductivity tensor in, for example, the $x$ direction is given by

$$
\sigma_{x x}=e^{2} \sum_{\nu \sigma} \int \frac{d \vec{q}}{8 \pi^{3}} \tau_{\nu \sigma}(\vec{q})\left|\hat{x} \cdot \vec{v}_{\nu \sigma}(\vec{q})\right|^{2} \delta\left(\varepsilon_{\nu \sigma}(\vec{q})-E_{F}\right),
$$

with $\tau_{\nu \sigma}(\vec{q})$ the relaxation time of state $\vec{q} \nu \sigma$. Oguchi ${ }^{34}$ and Zahn et $a l .{ }^{37}$ made the additional approximation that $\tau_{\nu \sigma}(\vec{q})$ does not depend on $\vec{q}$ and $\nu$. The spin-polarized conductivity can then be factorized into a scattering term and an electronic structure term, of which the latter can be evaluated without any free parameters. In the constant-relaxation-time approximation the electronic structure part of Eq. (8) for the diffusive conductivity resembles that of Eq. (2) for the ballistic conductance, except that the weighting over states is different by a factor $\left|\hat{x} \cdot \vec{v}_{\nu \sigma}(\vec{q})\right|$. We emphasize that the choice of a constant relaxation time is rather arbitrary. Equally well, the mean free path $l_{\nu \sigma}(\vec{q})=\tau_{\nu \sigma}(\vec{q})\left|\vec{v}_{\nu \sigma}(\vec{q})\right|$ could be chosen to be independent of $\vec{q}$ and $\nu$. The same electronic structure term as in the ballistic expression would be obtained by taking the mean free path in the $x$ direction constant in Eq. (8). For CPP transport this would correspond to a stateindependent mean free number of traversed interfaces. ${ }^{24}$ Note that in the calculation of the ballistic conductance such approximations are not required.

\section{MODEL CALCULATIONS}

Before evaluating the Sharvin conductance from first principles, it is instructive to first consider the results for several simple models.

\section{A. Free-electron mode}

The simplest model for the electronic structure is the freeelectron model with a single parabolic band. The Fermi surface of a free-electron gas in three dimensions is a sphere, the projections of the two hemispheres are circles with radius $k_{F}$, thus $S_{\sigma}(\hat{n})=2 \pi k_{F}^{2}$, independent of $\hat{n}$. By substitution of $S_{\sigma}(\hat{n})$ in Eq. (5) and using spin degeneracy the wellknown free-electron expression for the Sharvin conductance $G_{\mathrm{FE}}$ is obtained:

$$
G_{\mathrm{FE}}=\frac{2 e^{2}}{h} \frac{A k_{F}^{2}}{4 \pi} .
$$

Note the resemblance of $G_{\mathrm{FE}}$ to the electronic structure part $\sigma_{\text {Drude }}$ in Eq. (1).

In two dimensions the Fermi surface is a closed line in the two-dimensional Brillouin zone. For a free-electron gas the projection of the Fermi circle equals $4 k_{F}$ from which we obtain $^{57,58}$

$$
G_{\mathrm{FE}}^{2 \mathrm{D}}=\frac{2 e^{2}}{h} \frac{A k_{F}}{\pi},
$$

where the factor 2 comes from spin degeneracy.

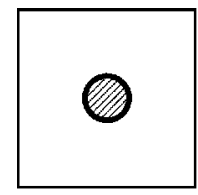

(a)

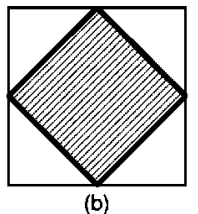

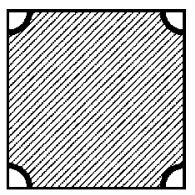

FIG. 1. The shape of the Fermi surface (thick line) for a nearestneighbor tight-binding model on a square lattice in two dimensions as a function of the band filling. The large squares are the boundaries of the first Brillouin zone, the hatched areas represent the filled states. (a) $\varepsilon_{0}-4 t \lesssim E_{F} \ll \varepsilon_{0} ; \quad$ (b) $E_{F}=\varepsilon_{0} ; \quad$ (c) $\varepsilon_{0} \ll E_{F} \lesssim \varepsilon_{0}$ $+4 t$.

\section{B. Tight-binding model}

In general the Sharvin conductance depends on the transport direction. This can be illustrated using a nearestneighbor tight-binding model for a square lattice in two dimensions. The dispersion relation depends on the on-site potential $\varepsilon_{0}$ and the hopping matrix element $t$ :

$$
\varepsilon_{\mathrm{TB}}^{2 \mathrm{D}}(\vec{q})=\varepsilon_{0}-2 t\left(\cos q_{x} a+\cos q_{y} a\right),
$$

where $a$ is the lattice parameter. The dispersion relation (11) determines the shape of the Fermi surface as a function of the band filling. When the Fermi energy is close to the bottom (top) of the band, the Fermi surface resembles the freeelectron (hole) Fermi circle, as shown schematically in Fig. 1(a) [Fig. 1(c)]. As $E_{F}$ approaches the center of the band, the Fermi surface starts to deviate from the free-electron behavior and exactly at half-filling it becomes a square, as shown in Fig. 1(b). The Fermi surface always has the fourfold symmetry of the underlying square lattice. From Fig. 1(b) it follows that the projection, and therefore the conductance, depends on $\hat{n}$; at half-filling the projection in the (10) direction is a factor $\sqrt{2}$ larger than the projection in the (11) direction. The Sharvin conductance is thus anisotropic even for a square lattice, in contrast to the diffuse conductivity. By analyzing the shape of the Fermi surface as a function of the band filling we obtain, for a spin-degenerate band,

$$
\begin{gathered}
G_{\mathrm{TB}}^{2 \mathrm{D}}(10)=\frac{2 e^{2}}{h} \frac{A}{a} \frac{1}{\pi} \arccos \left(\frac{\left|E_{F}-\varepsilon_{0}\right|}{2 t}-1\right), \\
G_{\mathrm{TB}}^{2 \mathrm{D}}(11)=\frac{2 e^{2}}{h} \frac{A}{a} \frac{\sqrt{2}}{\pi} \arccos \frac{\left|E_{F}-\varepsilon_{0}\right|}{4 t} .
\end{gathered}
$$

We used the fact that the boundaries of the projections along the (10) and the (11) directions are located on the lines $q_{y}=0$ and $q_{y}=q_{x}$, respectively, independent of the band filling. The numbers of conducting channels in the (10) and (11) directions are shown in Fig. 2 as a function of the band filling together with extrapolations of the free-electron and hole behavior near the top and bottom of the band, respectively.

For a nearest-neighbor tight-binding model on a cubic lattice in three dimensions the Fermi surface cannot be analyzed as easily as in the two-dimensional case. We therefore resort to numerical methods to calculate the projection. In Appendix A a suitable adaptation of the tetrahedron method $^{59-61}$ is described, including a discussion of its accuracy. This tetrahedron method will be used in the next sec- 


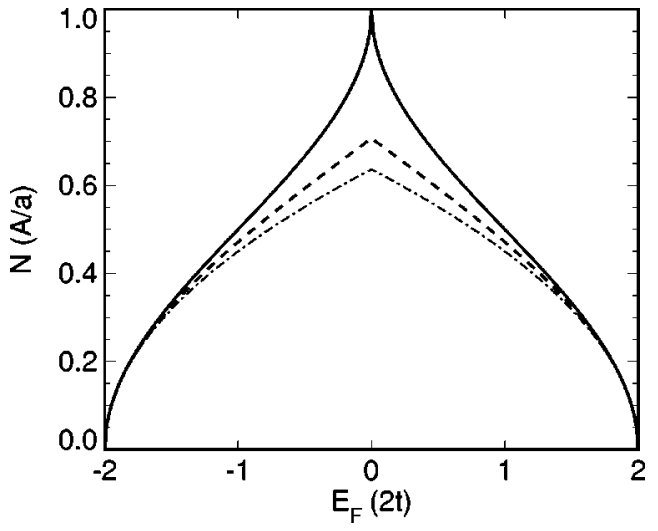

FIG. 2. The number of conducting channels per spin as a function of the Fermi energy for a nearest-neighbor tight-binding model on a square lattice in two dimensions. The on-site potential $\varepsilon_{0}$ is chosen as the zero of energy. The solid and the dashed lines are the numbers of conducting channels for transport in the (10) and (11) directions, respectively. The dashed-dotted line shows for energies smaller and larger than $\varepsilon_{0}$ the free-electron and the free-hole behavior, respectively.

tions in combination with first-principles band-structure calculations to evaluate the conductances of bulk materials and multilayers. For the three-dimensional (3D) tight-binding model, the numbers of conducting channels in the (100), (110), and (111) directions calculated numerically as well as the free-electron and hole extrapolations, from the top and bottom of the band, respectively, are shown in Fig. 3. The anisotropy in the Sharvin conductance is less pronounced than in the two-dimensional case. Our results for the (100) direction are in good agreement with the calculations by Todorov $^{62}$ except for the finite-size effects, which result from the finite cross section used in Ref. 62. In Appendix B an alternative calculation of the conductance in the (100) direction is given that agrees excellently with the result obtained using the tetrahedron method.

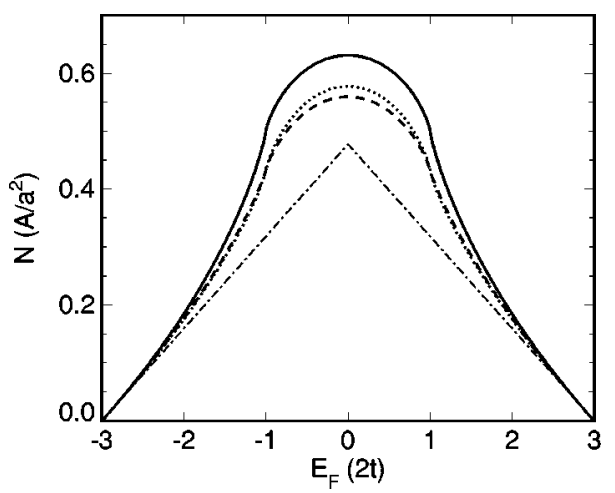

FIG. 3. The number of conducting channels per spin as a function of the Fermi energy for a nearest-neighbor tight-binding model on a cubic lattice in three dimensions. The on-site potential $\varepsilon_{0}$ is chosen as the zero of energy. The solid, dashed, and dotted lines are the numbers of conducting channels for transport in the (100), (110), and (111) directions, respectively. The dashed-dotted line shows for energies smaller and larger than $\varepsilon_{0}$ the free-electron and the free-hole behavior, respectively. The numbers of channels were calculated using $48^{3} k$ points in the first Brillouin zone.

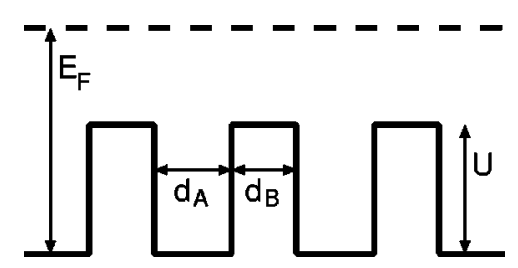

FIG. 4. The potential landscape for the Kronig-Penney model of a multilayer consisting of materials $A$ and $B$ with layer thicknesses $d_{A}$ and $d_{B}$, respectively. Only the situation that both materials are metallic is considered, i.e., that the potential step $U$ is smaller than the Fermi energy $E_{F}$.

\section{Kronig-Penney model}

The Kronig-Penney model has been used by several authors to study the effect of a modulated potential in the growth direction $z$ on the transport properties of magnetic multilayers. ${ }^{24,25,27-30,12}$ The effect of such a potential on the conductance can be significant in the CPP geometry and it is instructive to study this model in the ballistic limit. The potential landscape for the Kronig-Penney model is depicted in Fig. 4. First we consider the limit in which the potential is only a weak perturbation on the free-electron result. In Fig. 5(a) a cross section of the unperturbed Fermi sphere is plotted in an extended zone scheme. When the multilayer period $d_{A}+d_{B}$ is larger than half the Fermi wavelength $\lambda_{F}$, several Bragg planes cut the Fermi sphere. The perturbed Fermi surface will (almost always) intersect these Bragg planes perpendicularly, as is well known from nearly-free-electron theory. ${ }^{63}$ Gaps open in the projection of the Fermi surface in the $z$ or CPP direction, as shown in Fig. 5(b). This reduces the projected area and thus the conductance. The position of the gaps is determined by the multilayer period, their size by the strength of the potential. Near the edge of the projection the gaps become wider because electrons that meet the interfaces under grazing incidence can be reflected more easily. A similar nearly-free-electron analysis for the CIP directions shows that the opening of the gaps decreases the projection of the Fermi surface on one side of the Bragg planes, but increases it on the opposite side. These two contributions nearly cancel, causing only a small effect of the potential on the CIP conductance.

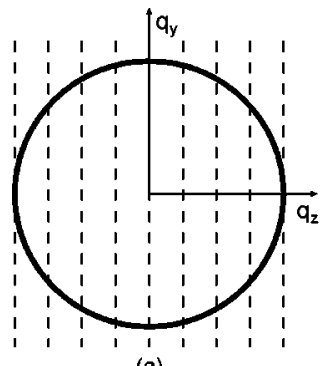

(a)

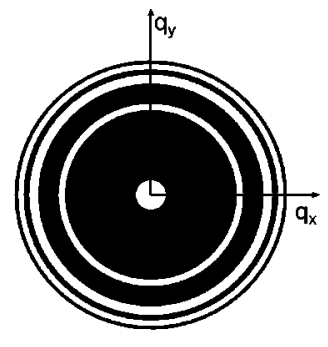

(b)
FIG. 5. (a) The cross section of the unperturbed Fermi sphere (thick line) at $q_{x}=0$. The spacing between the Bragg planes represented by the dashed lines equals $2 \pi /\left(d_{A}+d_{B}\right)$, which correspond to the multilayer period $d_{A}+d_{B}$ in the $z$ direction. (b) The projection (black area) of the perturbed Fermi surface in the $z$ or CPP direction. The positions of the gaps (white rings) in the perturbed Fermi surface correspond to the positions where the Bragg planes cut the unperturbed Fermi sphere. 

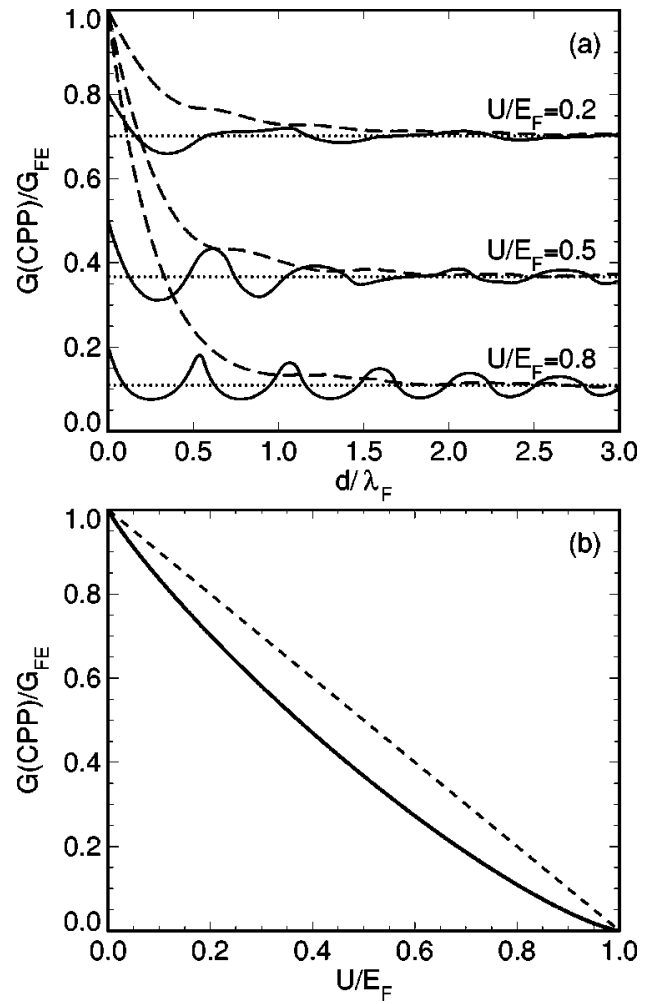

FIG. 6. (a) The Sharvin conductance in the $z$ or CPP direction for the Kronig-Penney model with $U / E_{F}=0.2,0.5$, or 0.8 as a function of the layer thicknesses $d_{A}$ (solid lines) and $d_{B}$ (dashed lines) with the other layer thickness constant and equal to $20 \lambda_{F}$. The dotted lines are the asymptotic values for $d_{A}, d_{B} \gg \lambda_{F}$. (b) The dependence of the Sharvin conductance for $d_{A}, d_{B} \gg \lambda_{F}$ on the height of the potential step. The dashed line represents the approximate result obtained by Bauer in Ref. 24 .

We study the ballistic conductance in the CPP direction $G(\mathrm{CPP})$, as a function of the layer thicknesses and the height of the potential step $U$. Using the cylindrical symmetry of the Fermi surface its projection can be calculated numerically by determining for $q_{\|}$from 0 to $k_{F}$ how many of the corresponding components of the Bloch vectors in the $z$ direction $q_{\perp}$ are real and correspond to a conducting channel. Figure 6(a) shows the dependence of $G(\mathrm{CPP})$ on the layer thicknesses $d_{A}$ and $d_{B}$ for a constant value of $U$. For $d_{B}=0$ the free electron result is retrieved. For $d_{A}=0$ the bulk conductance of material $B$ is obtained that is only $\left(1-U / E_{F}\right)$ times $G_{\mathrm{FE}}$ due to the reduction of the Fermi wave vector in the bulk of material $B$ by the potential step. ${ }^{24}$ For large layer thicknesses the number of gaps increases but their sizes become smaller and $G(\mathrm{CPP})$ converges to some finite value that is lower than either of the bulk conductances. The dependence of this asymptotic value of $G(\mathrm{CPP})$ on $U$ is shown in Fig. 6(b) and derived in Appendix C. The main reduction of $G(\mathrm{CPP})$ comes from states $\vec{q}_{\|}$that are propagating in material $A$ but evanescent in material $B .^{24}$ The additional reduction of $G(\mathrm{CPP})$ is due to the perturbation by the potential of the states that are propagating in both materials. The contribution of the states that are evanescent in material $B$ decreases exponentially with $d_{B}$.

For finite values of the layer thicknesses, oscillations arise from the interference of the propagating states between dif-

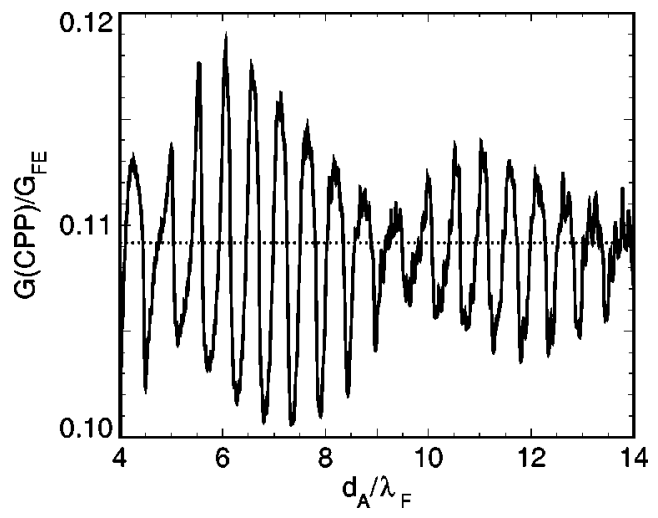

FIG. 7. The oscillations of the ballistic conductance as a function of $d_{A}$ around the asymptotic value (dotted line) for the KronigPenney model with $U / E_{F}=0.8$ and $d_{B}=20 \lambda_{F}$.

ferent interfaces [see Fig. 6(a)]. Mathon, Villeret, and Itoh ${ }^{64}$ studied similar oscillations in the transmission through trilayers. From the approach used in Appendix $\mathrm{C}$ it is obvious that the ballistic conductance of a multilayer is intimately related to the transmission through a single barrier, i.e., a trilayer. In Fig. 7 the dependence of $G(\mathrm{CPP})$ on $d_{A}$ is plotted for $U / E_{F}=0.8$ for large layer thicknesses. In Ref. 64 two asymptotic oscillation periods are identified: one from the stationary point of the Fermi surface $\left(\lambda_{F} / 2\right)$ and the other from the cutoff point due to the potential step that is equal to $\left(\lambda_{F} / 2\right)\left(E_{F} / U\right)$. The oscillations shown in Fig. 7 correspond very well with these two oscillation periods. This is another manifestation of the close relationship between the single barrier scattering properties and the ballistic conductance of the multilayer. Figure 7 also shows that the asymptotic value obtained from Eq. (C8) and plotted in Fig. 6(b) corresponds very well with the value around which the numerical results oscillate for large but finite layer thicknesses.

\section{BULK MATERIALS}

We now turn to the calculation of the ballistic conductance for real materials. To obtain a realistic description of the electronic structure that does not contain any free parameters we calculate band structures in the local-spin-density approximation (LSDA). The only input for such calculations is the species and the positions of the atoms. The LSDA provides an accurate, though not exact, description of Fermi surfaces. ${ }^{65,66}$ Note that the conductance as an integrated property is not very sensitive to the details of the Fermi surface topology. We make use of the linear-muffin-tin orbital (LMTO) method in the atomic-spheres approximation (ASA). ${ }^{67,68}$ For the bulk calculations presented in this section the electronic structure was calculated self-consistently using a mesh of $12^{3} k$ points in the first Brillouin zone. Unless stated otherwise a basis of $s, p$, and $d$ orbitals is used; the effect of $f$ states is discussed below. To evaluate the projection of the Fermi surfaces use is made of the tetrahedron method described in Appendix A. Good convergence was obtained using interpolation grids with as many as $48^{3} k$ points in the first Brillouin zone. 


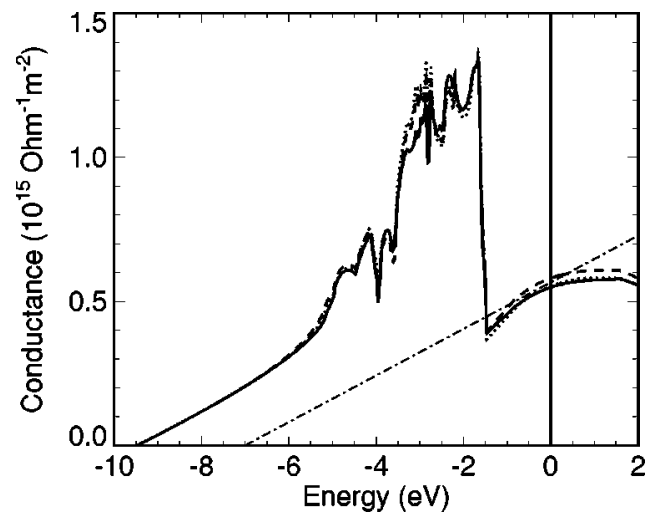

FIG. 8. The ballistic conductance per spin for bulk fcc copper as a function of the energy for a rigid band structure. The Fermi energy is chosen as the zero of energy. The solid, dashed, and dotted lines are the Sharvin conductances for transport in the (100), (110), and (111) directions, respectively. The conductances were calculated using $48^{3} k$ points in the first Brillouin zone. The dasheddotted line shows the free electron result obtained using Eq. (9) and the parameters from Ref. 63.

Figure 8 shows the ballistic conductance of bulk $\mathrm{Cu}$ for three different transport directions. The Sharvin conductance depends on $\hat{n}$ but the anisotropy is small compared to that in the tight-binding model. This can partly be explained by the close-packed structure of the face-centered-cubic crystal in which each atom has 12 nearest-neighbor atoms compared to 6 nearest-neighbor atoms in the more open simple-cubic lattice considered in the model calculation. To indicate the contributions from different bands the conductance is plotted as a function of the energy keeping the $\mathrm{Cu}$ band structure unmodified. Obviously, only the value at the Fermi energy is relevant for the linear response regime. The free-electron behavior of Eq. (9) is also shown, assuming a density of one free electron per $\mathrm{Cu}$ atom. ${ }^{63}$ Around the Fermi energy the ballistic conductance corresponds very well with the freeelectron estimate of $0.57 \times 10^{15} \Omega^{-1} \mathrm{~m}^{-2}$ at $E_{F}$. For energies around $3 \mathrm{eV}$ below the Fermi energy the ballistic conductance is significantly larger due to the high density of electrons of mainly $d$ character. The enhancement is, however, considerably less pronounced than in the corresponding density of states plot ${ }^{69}$ (not shown) due to the weighting in Eqs. (4) and (5) with the velocity, which is much smaller for the relatively flat bands in this energy range. For energies more than $6 \mathrm{eV}$ below the Fermi energy there is only one band that has mainly $s$ character. The Sharvin conductance in this regime is shifted in energy with respect to the freeelectron line but has the same slope, which means that the curvature of this band corresponds to the free-electron mass.

In magnetic materials the degeneracy between the majority and the minority spin electrons is broken. The ballistic conductance then depends on the spin direction due to the spin dependence of the electronic structure. In Fig. 9 the Sharvin conductance is shown for bulk fcc Co. For the majority spin the dependence of the conductance on the band filling resembles that of $\mathrm{Cu}$, except that the width of the bands with mainly $d$ character is larger. The calculated (spin) magnetization of Co is 1.63 Bohr magneton $\left(\mu_{B}\right)$ per atom, which yields an estimate for the density of free majority-spin electrons of 0.32 per atom. The corresponding free electron

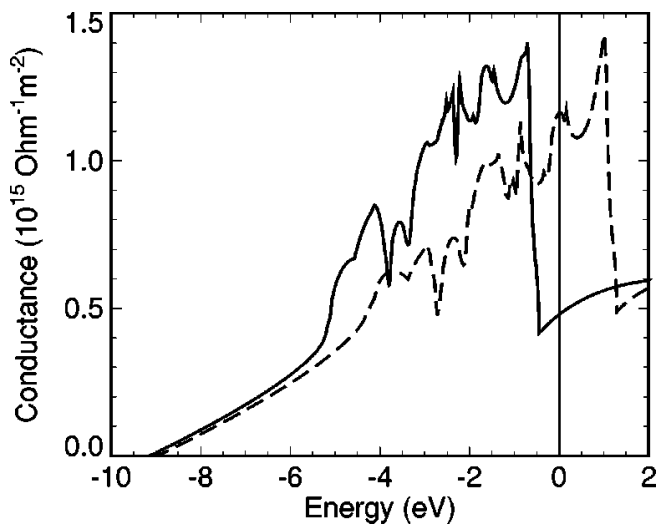

FIG. 9. The ballistic conductance for bulk fcc cobalt as a function of the energy for a rigid band structure. The Fermi energy is chosen as the zero of energy. The solid and dashed lines are the Sharvin conductances for transport in the (100) direction for the majority and minority spin electrons, respectively. The conductances were calculated using $48^{3} k$ points in the first Brillouin zone.

estimate for the ballistic conductance is $0.43 \times 10^{15}$ $\Omega^{-1} \mathrm{~m}^{-2}$, which is only about $10 \%$ smaller than the calculated value in Fig. 9. For the minority spin the $d$-like bands are shifted to higher energies. At the Fermi energy this results in a minority spin conductance which is more than twice the majority spin conductance. In contrast to the results for $\mathrm{Cu}$ and the majority spin of $\mathrm{Co}$, no reasonable freeelectron estimate can be given for the minority-spin conductance of $\mathrm{Co}$, which reflects the complicated band structure for the Co minority spin around the Fermi energy.

In the expansion of the wave function only a limited number of partial waves are taken into account. To estimate the influence of the cutoff in angular momentum we compare the Sharvin conductances at $E_{F}$ for $\mathrm{Cu}$ and Co calculated using spd and $s p d f$ bases. As shown in Table I the difference is

TABLE I. The Sharvin conductances per spin (in units $10^{15} \Omega^{-1} \mathrm{~m}^{-2}$ ) in the (100), (110), and (111) directions for a number of cubic metals. Most calculations are performed using an $s p d$ basis and for the experimental lattice constant $a$ which is taken from Ref. 68. For $\mathrm{Co}$ and $\mathrm{Cu}$ results obtained using an $s p d f$ basis are also listed, as well as the conductance for $\mathrm{Cu}$ using the experimental lattice constant of Co and vice versa.

\begin{tabular}{lcccccc}
\hline \hline & $a(\AA)$ & basis & spin & $(100)$ & $(110)$ & $(111)$ \\
\hline fcc $\mathrm{Cu}$ & 3.614 & spd & & 0.55 & 0.58 & 0.55 \\
fcc Cu & 3.614 & spdf & & 0.55 & 0.58 & 0.55 \\
fcc Cu & 3.549 & spd & & 0.57 & 0.60 & 0.57 \\
fcc Co & 3.549 & spd & maj & 0.48 & 0.49 & 0.45 \\
fcc Co & 3.549 & spdf & maj & 0.47 & 0.47 & 0.43 \\
fcc Co & 3.614 & spd & maj & 0.47 & 0.49 & 0.45 \\
fcc Co & 3.549 & spd & min & 1.16 & 1.09 & 1.10 \\
fcc Co & 3.549 & spdf & min & 1.19 & 1.11 & 1.13 \\
fcc Co & 3.614 & spd & min & 1.12 & 1.05 & 1.06 \\
fcc Ni & 3.523 & spd & maj & 0.48 & 0.49 & 0.45 \\
fcc Ni & 3.523 & spd & min & 1.38 & 1.36 & 1.34 \\
bcc Fe & 2.861 & spd & maj & 0.48 & 0.42 & 0.43 \\
bcc Fe & 2.861 & spd & min & 0.92 & 0.89 & 0.93 \\
bcc Cr & 2.885 & spd & & 0.63 & 0.57 & 0.59 \\
\hline \hline
\end{tabular}


negligible for $\mathrm{Cu}$, the largest deviation being $0.003 \times 10^{15} \Omega^{-1} \mathrm{~m}^{-2}$ for the (100) direction. For Co the differences are significantly larger but do not exceed $4 \%$. We attribute this deviation mainly to the small change in the magnetic moment of Co. The magnetization of Co decreases from 1.63 to $1.59 \mu_{B}$ /atom when $f$ orbitals are included. This gives rise to a decrease of $6 \%$ in the estimated density of free electrons in the majority-spin band, which corresponds to a decrease of $4 \%$ in the Sharvin conductance, in reasonable agreement with the calculated values for the Co majority spin.

In multilayers the layers of the constituent bulk materials are strained. We therefore evaluate the dependence of the Sharvin conductance on the atomic volume by performing calculations for $\mathrm{Cu}$ using the experimental lattice constant of $\mathrm{Co}$ and vice versa (see Table I). For $\mathrm{Cu}$ the increase in the conductance for a smaller lattice parameter can be understood in terms of the free-electron model. From Eq. (9) we expect an enhancement of $3.7 \%$ in the Sharvin conductance when the lattice constant decreases by $1.8 \%$. The calculated enhancements for $\mathrm{Cu}$ are $3.7 \%, 3.5 \%$, and $3.3 \%$ for the (100), (110), and (111) directions, respectively. For Co at the lattice parameter of $\mathrm{Cu}$ the magnetization is increased to $1.67 \mu_{B}$ /atom, which is due to the reduced overlap of atomic orbitals. The reduction of the conductance for the majority spin due to the larger lattice parameter is almost exactly canceled by the enhancement due to the larger magnetic moment. For $\mathrm{Cu}$ and for either spin of Co the changes in the conductance due to the $1.8 \%$ change in the lattice parameter are smaller than $4 \%$.

The calculations can be straightforwardly carried out for other materials. Besides the results for $\mathrm{Cu}$ and $\mathrm{Co}$, Table I also displays the Sharvin conductances at $E_{F}$ for fcc $\mathrm{Ni}$ and bcc $\mathrm{Fe}$ and $\mathrm{Cr}$.

\section{Co/Cu MULTILAYERS}

The method of calculation for multilayers is similar to that for bulk materials in the previous section. Using the LMTO method in the ASA the band structures of magnetic multilayers are calculated for both parallel $(\mathrm{P})$ and antiparallel (AP) alignment of the magnetizations in adjacent magnetic layers that are separated by nonmagnetic layers. This section focuses on (100) oriented $\mathrm{Co}_{n} / \mathrm{Cu}_{n}$ multilayers, where $n$ is the number of atomic layers of each material in a unit cell that is repeated periodically in the growth direction. Results are also obtained for a $\mathrm{Co}_{3} / \mathrm{Cu}_{3}$ multilayer in the (111) orientation.

\section{A. Technical aspects}

The lattice distortion that arises from the $1.8 \%$ lattice mismatch between $\mathrm{Co}$ and $\mathrm{Cu}$ is taken into account using the model from Ref. 70, which was based upon the results of total-energy calculations: the in-plane lattice parameter is chosen to be that of bulk fcc copper, the cobalt layer is tetragonally distorted keeping the bond lengths between nearest-neighbor atoms constant. The lattice constants in the calculations are chosen to be $1 \%$ smaller than the experimental ones in order to be close to the total-energy minimum for calculations in the local-density approximation; the bulk lattice parameter of $\mathrm{Cu}$ is thus taken to be $3.578 \AA$.
The charge and spin densities in the $\mathrm{P}$ configurations are calculated self-consistently using a basis of $s, p, d$, and $f$ orbitals and meshes of approximately $1400 k$ points in the first Brillouin zone. From the self-consistent potentials the band structures are determined using an $s p d$ basis and different meshes containing up to $55000 k$ points in the first Brillouin zone. The projections of the Fermi surfaces are then calculated by the tetrahedron method described in Appendix A. For $\mathrm{Co}_{4} / \mathrm{Cu}_{4}$ in the $\mathrm{P}$ configuration we performed the self-consistent calculations of the charge and spin densities both using an $s p d$ and using an $s p d f$ basis. The conductances (calculated with an $s p d$ basis) differed by less than $3 \%$.

The band structures in the AP configurations are calculated from potentials obtained by interchanging the spin densities from the corresponding $\mathrm{P}$ configurations on alternating $\mathrm{Co}_{n} / \mathrm{Cu}_{n}$ cells. By calculating the potentials in the AP configuration for $n=1$ and $n=2$ self-consistently, we checked that this procedure has only a minor effect on the final results: for $n=2$ the difference in the magnetic moment on the Co atoms is as small as $0.01 \mu_{B}$ and the relative changes in the conductances do not exceed $1 \%$. When $n$ is odd the charge density on the $\mathrm{Cu}$ atom in the center of the layer in the AP configuration is taken equal to the charge density of bulk $\mathrm{Cu}$. Alternatively, the average of the charge densities for the majority and minority spins in the $\mathrm{P}$ configuration can be used; for $\mathrm{Co}_{3} / \mathrm{Cu}_{3}$ the conductances in the AP configuration that follow from these two choices differ by approximately $1 \%$.

From the above discussion it is clear that there are several sources of (minor) inaccuracy. Fortunately, the conductance is not very sensitive to the precise parameters used in the calculation, changing typically by a few percent when individual parameters are changed. The overall error bars of the conductances are estimated to be smaller than $10 \%$. This is sufficiently accurate for our present study in which we are interested in changes in the conductance that are large (giant). In the following we will quote the calculated conductances without error bars.

\section{B. (100) orientation}

Figures 10(a) and 10(b) show the projections of the Fermi surfaces in the CPP direction for the two spins of a (100) oriented $\mathrm{Co}_{5} / \mathrm{Cu}_{5}$ multilayer in the $\mathrm{P}$ configuration. The total black area is a measure of the conductance although it should be noted that different sheets of Fermi surface might be projected on top of each other, which is not visible in the figures but which is taken into account in the numerical results. The projection for the majority spin resembles the free-electron projection, which is a circle. As in Fig. 5(b) for the KronigPenney model the projection is split up into a series of rings separated by minigaps (the "white" rings) due to the presence of a periodic multilayer potential. Furthermore, the free-electron circle is distorted in a way that reflects the fourfold symmetry of the underlying lattice. This is similar to the effect found for the tight-binding model in Fig. 1(b). The projection for the minority spin is more difficult to interpret and certainly not free-electron like, which is caused by the complicated Fermi surface of the Co minority-spin electrons. It is clear that the total projected area and thus the number of 


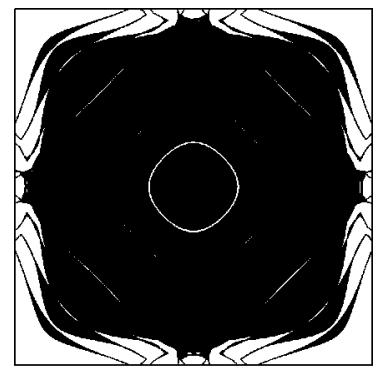

(a)

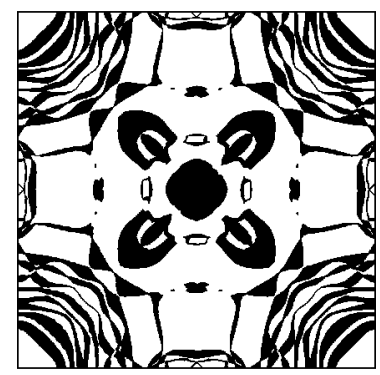

(b)

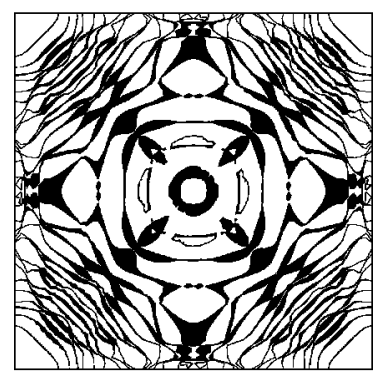

(c)

FIG. 10. Projections inside the first Brillouin zone of different Fermi surfaces for a (100) oriented $\mathrm{Co}_{5} / \mathrm{Cu}_{5}$ multilayer on a plane parallel to the interfaces. (a) Majority spin and (b) minority spin in the parallel configuration; (c) either spin in the antiparallel configuration.

channels available for conduction is much smaller than for the majority spin. In the language of the Kronig-Penney model this corresponds to a higher potential step. Some states (the thin lines) have no dispersion in the direction normal to the multilayer planes and can be identified as quantum-well states. Because their velocity is perpendicular to $\hat{n}$, their contribution to the CPP transport is negligible. Figure 10(c) shows the projection of the Fermi surface of the same multilayer in the AP configuration. Because the projections for spin-up and spin-down electrons are identical, only one is shown. The projection in the AP configuration is more similar to the projection for the minority spin than to that for the majority spin in the $\mathrm{P}$ configuration. The number of gaps is, however, twice as large for the AP configuration because the unit cell is doubled. The magnetoresistance is defined as

$$
M R=\frac{G_{\mathrm{maj}}+G_{\mathrm{min}}-2 G_{\mathrm{AP}}}{2 G_{\mathrm{AP}}},
$$

with $G_{\text {maj }}$ and $G_{\text {min }}$ the conductances in the P configuration for the majority- and the minority-spin electrons, respectively, and $G_{\mathrm{AP}}$ the conductance of either spin in the AP configuration. The CPP MR for the $\mathrm{Co}_{5} / \mathrm{Cu}_{5}$ multilayer of Fig. 10 is $120 \%$. We thus predict the unexpected result that

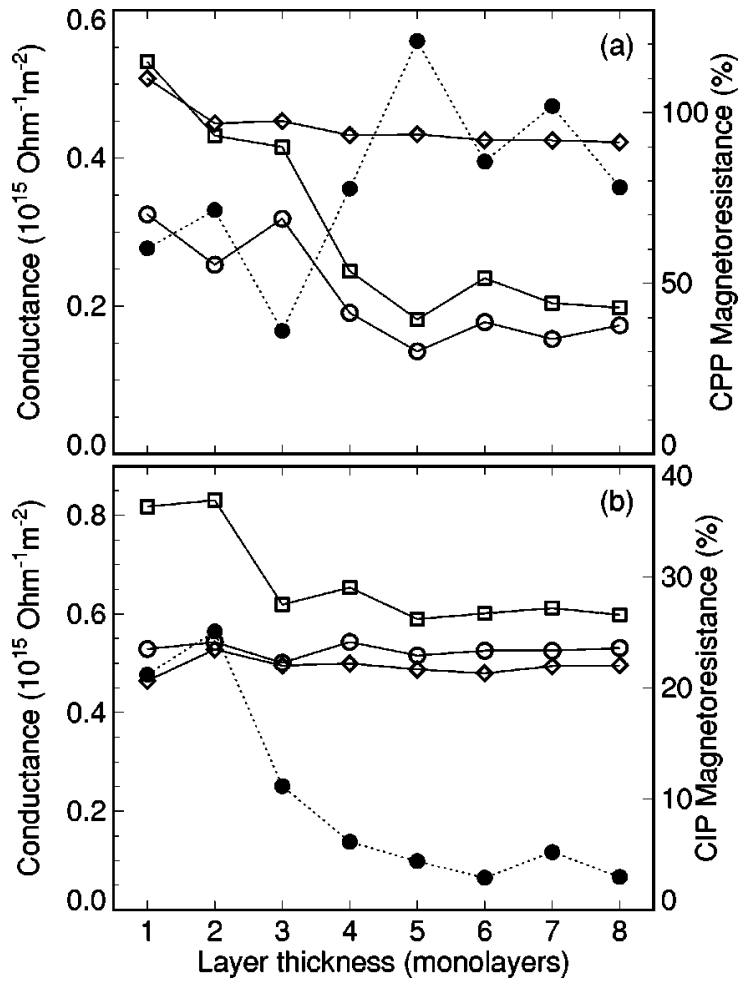

FIG. 11. The layer thickness dependences for (100) oriented $\mathrm{Co}_{n} / \mathrm{Cu}_{n}$ multilayers of the MR (filled circles) and of the conductances for the majority (diamonds) and the minority (squares) spin in the parallel configuration and for both spins in the antiparallel configuration (open circles). (a) CPP geometry, (b) CIP geometry.

transport measurements in the ballistic regime will find values for the CPP MR comparable to those measured in the diffusive limit. ${ }^{71,72}$

Figure 11 summarizes the results for layer thicknesses varying from $n=1-8$. The thickness dependences in the CPP geometry can be interpreted in terms of the KronigPenney model, compare Figs. 6(a) and 11(a). For small layer thicknesses the conductances decrease rapidly with $n$, which arises at least partly from states that are evanescent in either $\mathrm{Co}$ or $\mathrm{Cu}$. An additional contribution to the enhanced conductances at small $n$ might originate from the deviations of the potentials near the interfaces from the bulk potentials, which can smooth the discontinuity and modify the height of the potential step for thin layers. The magnetic moments on the central Co atoms, for example, are, for $n=1-4$, slightly reduced compared to the bulk value (by at most $0.2 \mu_{B}$ ). For larger layer thicknesses the conductances approach a constant value. Small oscillations of the conductance as a function of the number of monolayers are observed for both spins in the AP configuration and for the $\mathrm{P}$ minority spin, reflecting quantum size effects on the conductance. Qualitatively, the most important effect for the CPP geometry can be cast in terms of the "semiclassical" model of Ref. 24: the resistance for the minority-spin channel in the P configuration is approximately equal to that of either channel in the AP configuration but is shunted by the "open" majority-spin channel. Quantitatively, however, the present realistic calculations yield much larger results for the magnetoresistance than could be expected on the basis of the simple estimates made in Ref. 24. 
For the CIP geometry it is not very instructive to plot the Fermi surface projection because many sheets of Fermi surface are projected on top of each other due to the reduced size of the Brillouin zone in the $z$ direction. The numerical calculations are, however, straightforward and the results are shown in Fig. 11(b). Unless stated otherwise our calculations for the CIP geometry are for the current along a (100) direction, results along a (110) direction being very similar (see below). As in the CPP case the conductances saturate for large layer thicknesses. For the majority spin in the P configuration the conductance is approximately the average of the bulk conductances of $\mathrm{Cu}$ and $\mathrm{Co}$. This corresponds to the simple interpretation of two layers contributing to the conductance in parallel. For the minority spin the multilayer conductance is considerably smaller than the average of the bulk conductances and the simple interpretation breaks down. The conductance of either spin in the AP configuration is approximately the average of $G_{\text {maj }}$ and $G_{\text {min }}$. The calculated CIP MR of $\mathrm{Co} / \mathrm{Cu}$ is only a few percent, which is much smaller than the values that are observed experimentally in the diffusive regime. ${ }^{73}$

The breakdown of the simple interpretation for the minority-spin conductance can be understood as follows. In the limit of thick layers the Bloch states of an $A / B$ multilayer are linear combinations of bulk Bloch states of materials $A$ and $B$. The velocity $\vec{v}_{i}$ of multilayer Bloch state $i$ at $\vec{q}_{\|}$and $E_{F}$ can then be written in terms of the velocities $\vec{v}_{j}^{A}$ and $\vec{v}_{k}^{B}$ of the bulk Bloch states $j$ and $k$ in materials $A$ and $B$, respectively, at $\vec{q}_{\|}$and $E_{F}$ as

$$
\vec{v}_{i}=\sum_{j}\left|\alpha_{i j}\right|^{2} \vec{v}_{j}^{A}+\sum_{k}\left|\beta_{i k}\right|^{2} \vec{v}_{k}^{B},
$$

where $\alpha_{i j}$ and $\beta_{i k}$ are the coefficients of the linear expansion. The local density of states for a multilayer in the thick layer limit is the same as in the bulk from which we obtain the relations

$$
\begin{aligned}
& \sum_{i}\left|\alpha_{i j}\right|^{2}=\frac{d_{A}}{d_{A}+d_{B}}, \\
& \sum_{i}\left|\beta_{i k}\right|^{2}=\frac{d_{B}}{d_{A}+d_{B}} .
\end{aligned}
$$

For the calculation of the conductance using Eq. (2) the sum of the absolute values of the velocities in direction $\hat{n}$ is needed. Employing Eqs. (14) and (15) and the relation $\left|\Sigma_{i} x_{i}\right| \leqslant \Sigma_{i}\left|x_{i}\right|$ yields

$$
\sum_{i}\left|\hat{n} \cdot \vec{v}_{i}\right| \leqslant \frac{d_{A}}{d_{A}+d_{B}} \sum_{j}\left|\hat{n} \cdot \vec{v}_{j}^{A}\right|+\frac{d_{B}}{d_{A}+d_{B}} \sum_{k}\left|\hat{n} \cdot \vec{v}_{k}^{B}\right| .
$$

The equality in Eq. (16) only holds if all velocity components $\hat{n} \cdot \vec{v}_{j}^{A}$ and $\hat{n} \cdot \vec{v}_{k}^{B}$ have the same sign. This condition is fulfilled in the CIP geometry for the majority spin of $\mathrm{Co} / \mathrm{Cu}$ multilayers and the multilayer conductance is the average of the bulk conductances weighted by the layer thicknesses. A similar point was made for the diffusive conductivity in the Kronig-Penney model by Levy. ${ }^{12}$ The argument breaks

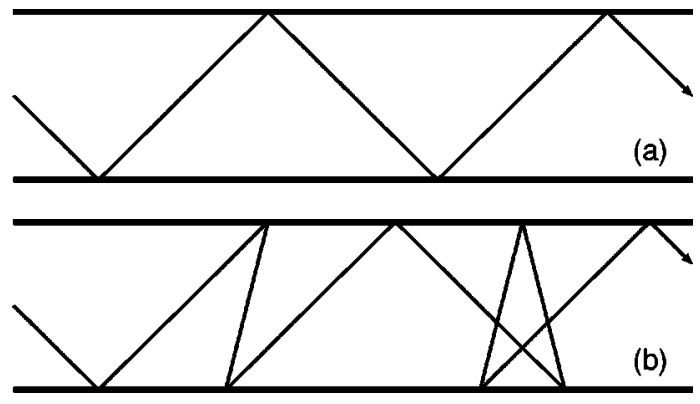

FIG. 12. Schematic representation of a single scattering path of a quantum-well state for (a) a single sheet and (b) two sheets of Fermi surface. In (b) the states at different sheets have opposite velocity components along the interfaces. There is no transmission through the interfaces that are indicated by the thick black lines.

down, however, if not all velocity components in direction $\hat{n}$ have the same sign. In that case the inequality in Eq. (16) applies and the multilayer conductance is reduced compared to the averaged bulk conductances. This is consistent with our results for the minority spin. Weissmann et al. ${ }^{46}$ find for $\mathrm{Co} / \mathrm{Ni}$ multilayers in the CIP geometry that the multilayer conductance is approximately equal to the weighted average of the bulk conductances for the majority spin whereas it is considerably smaller for the minority-spin conductance; this result is also consistent with our interpretation.

The physical origin of the reduction of the multilayer conductance in the CIP geometry is elucidated in Fig. 12 for a state that is localized in one layer. In Fig. 12(a) there is only one sheet of Fermi surface and the two bulk Bloch states with energy $E_{F}$ and wave vector $\vec{q}=\left(\vec{q}_{\|}, \pm q_{\perp}\right)$ have velocity components that are the same in the CIP but opposite in the CPP direction. In this case the velocity in the CIP direction is not altered by the reflection at the interfaces. In Fig. 12(b) there are two sheets of Fermi surface and states can be reflected into states on the other sheet of the Fermi surface that have an opposite velocity component along the interfaces. Due to the mixing by the intersheet scattering the multilayer Bloch states are linear combinations of bulk states from the two different sheets, which slows down the multilayer Bloch electrons and thereby reduces the conductance. The reduction of the multilayer conductance in the CIP geometry in this case thus originates from reflection, which changes the velocity direction along the interface, even though the reflection is "specular" in the sense that $\vec{q}_{\|}$is conserved. In the CPP geometry, reduction of the multilayer conductance occurs even in the case of a single sheet of Fermi surface because the two states at $\vec{q}_{\|}$have opposite velocity components perpendicular to the interfaces.

In principle, the intersheet scattering in magnetic multilayers depends on the magnetic configuration and can thereby give rise to a finite CIP MR. From the low values obtained for the CIP MR we conclude that this mechanism is not very effective in $\mathrm{Co} / \mathrm{Cu}$. This probably indicates that the intersheet scattering occurs predominantly by reflection of states in the Co minority layers and that this reflection hardly depends on the potential landscape beyond the directly adjacent $\mathrm{Cu}$ layers. For $\mathrm{Fe} / \mathrm{Cr}$ multilayers the mechanism of intersheet scattering turns out to be more efficient in generating a CIP MR and we will elaborate on this in Sec. VI. 


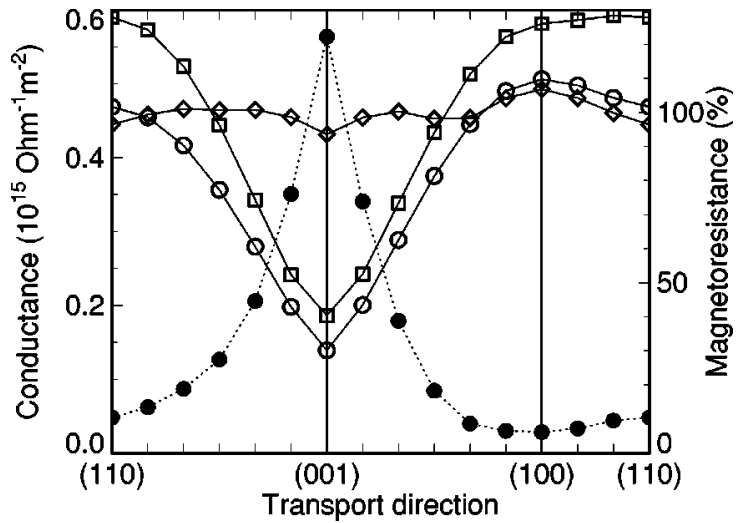

FIG. 13. The dependences on the direction of transport for a (100) oriented $\mathrm{Co}_{5} / \mathrm{Cu}_{5}$ multilayer of the MR (filled circles) and of the conductances for the majority (diamonds) and the minority (squares) spin in the parallel configuration and for both spins in the antiparallel configuration (open circles). The CPP geometry corresponds to the (001) direction, (100) and (110) are two CIP directions. The spacing between adjacent tick marks on the horizontal axis corresponds to a rotation of the transport direction by $15^{\circ}$. The conductances were calculated using $72 \times 72 \times 12 k$ points in the first Brillouin zone.

Besides for the CPP and CIP geometries the conductance can also be calculated for the CAP geometry ${ }^{19}$ in which the current flows at an angle to the interface planes. Figure 13 shows for a (100) oriented $\mathrm{Co}_{5} / \mathrm{Cu}_{5}$ multilayer the dependences of the conductances and the MR on $\hat{n}$. The majorityspin conductance is only weakly dependent on the transport direction as expected. The dependences of $G_{\min }$ and $G_{\mathrm{AP}}$ can roughly be described by a constant term plus a term proportional to $|\sin \theta|$ with $\theta$ the angle between $\hat{n}$ and the $(001)$ or CPP direction. The constant term can be interpreted as the projection of spherical parts of the Fermi surface representing propagating states. The $|\sin \theta|$ term then arises from parts of the Fermi surface that have their normals perpendicular to the (001) direction, which is characteristic for quantum-well states. In reality such a strict division between propagating and quantum-well states is too crude, of course. The conductances and the MR depend only weakly on the CIP direction, the main anisotropy in multilayers is thus between CPP and CIP.

\section{C. $s p$ - $d$ hybridization}

The large value we calculate for the CPP MR is in striking contrast to what one would expect on the basis of simple models that ascribe the GMR effect completely to spindependent scattering at defects. In the simplest free-electron models potential steps are absent and the electronic structure does not depend on the magnetic configuration at all. In the ballistic regime where defect scattering is absent the MR is then expected to vanish. To understand the origin of the discrepancy between the simple models and the realistic calculations we investigate the effect of hybridization between the $d$ electrons and the free electrons in the $s p$ band. This hybridization is neglected in most early theories. ${ }^{20-33}$ In our calculations we switch the $s p$ - $d$ hybridization off by setting the matrix elements between the $s p$ and the $d$ orbitals in the LMTO structure constants equal to zero, but keeping the

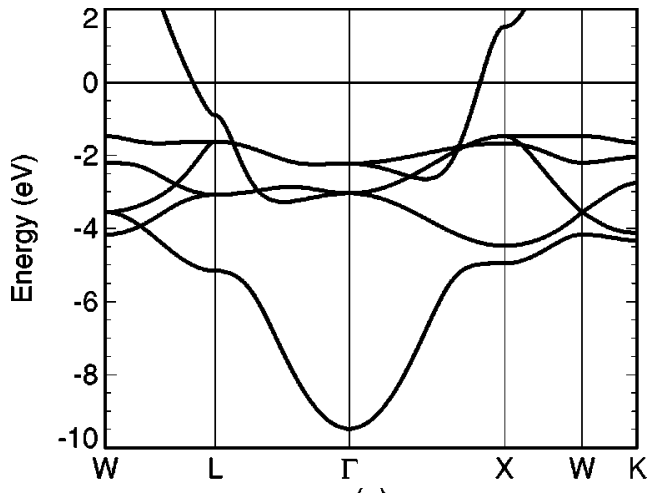

(a)

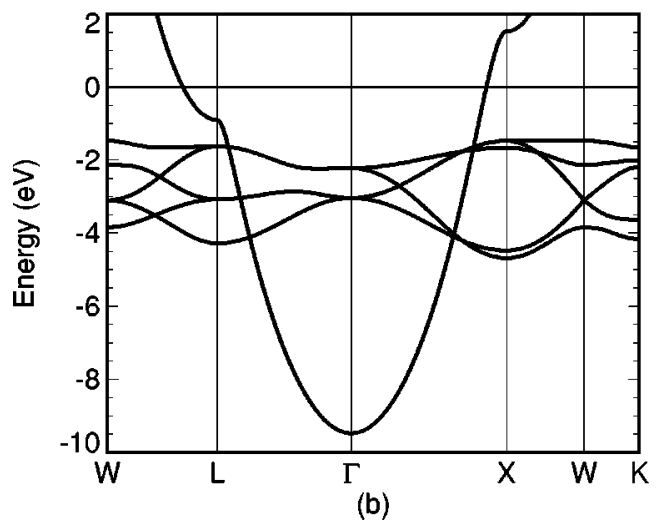

FIG. 14. The band structures of bulk $\mathrm{Cu}$ (a) with and (b) without $s p-d$ hybridization. The Fermi energy of the hybridized bands is chosen as the zero of energy.

potentials unchanged. The band structure for bulk $\mathrm{Cu}$ without $s p$ - $d$ hybridization, as shown in Fig. 14(b), mimics the electronic structure adopted in simple models: a single nearly parabolic band crossing localized $d$ bands without mixing. In contrast, the correct calculation that includes $s p$ - $d$ hybridization displays strong anticrossing of different bands, as shown in Fig. 14(a). For $\mathrm{Cu}$ the difference in electronic structure around the Fermi energy is small, and the same is true for the majority spin in Co. For the minority spin, however, the Fermi energy lies in the $d$ bands where the modifications in the band structure due to hybridization are substantial.

The projections of the Fermi surfaces for a (100) oriented $\mathrm{Co}_{5} / \mathrm{Cu}_{5}$ multilayer without $s p$ - $d$ hybridization in Fig. 15 are strikingly different from the projections with $s p-d$ hybridization in Fig. 10. The projections of the $s p$ parts of the unhybridized Fermi surfaces are free-electron like, with only small distortions due to the fourfold rotational symmetry. Because the diameters of the circles are greater than the width of the first Brillouin zone the Fermi surfaces are folded back at the zone boundaries. The minigaps formed by the periodic multilayer potential are small but clearly resolved. For the minority spin in the $\mathrm{P}$ configuration and for both spins in the AP configuration there are also $d$ states present at $E_{F}$. These bands are confined to a single magnetic layer and have only small widths in the direction perpendicular to the interfaces. These quantum-well states hardly contribute to transport perpendicular to the interfaces. The positions of the quantum-well states are the same in Figs. 15(b) and 15(c) because the width of the wells is the same. A careful analysis shows that the lines in Fig. 15(c) are slightly narrower, 


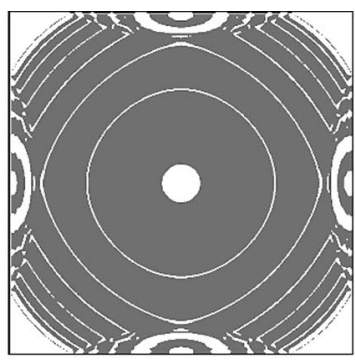

(a)

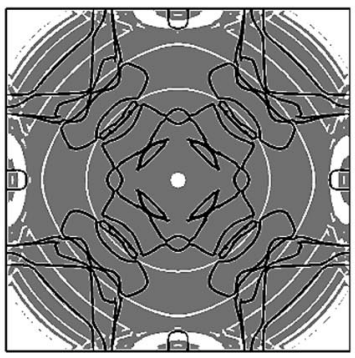

(b)

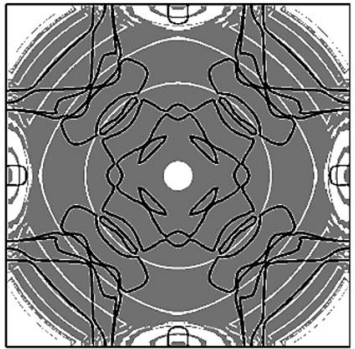

(c)

FIG. 15. Projections inside the first Brillouin zone of different Fermi surfaces for a (100) oriented $\mathrm{Co}_{5} / \mathrm{Cu}_{5}$ multilayer on a plane parallel to the interfaces. In the calculations the $s p-d$ hybridization is omitted. The $s p$ parts of the Fermi surface are gray and the $d$ parts are black. (a) Majority spin and (b) minority spin in the parallel configuration; (c) either spin in the antiparallel configuration.

which is due to the larger interwell spacing in the AP configuration. The MR decreases from $120 \%$ to $3 \%$ when the $s p-d$ hybridization is discarded. A similar calculation for $\mathrm{Co}_{4} / \mathrm{Cu}_{4}$ yields a reduction from $78 \%$ to $1 \%$. Such low values were already estimated from a simple model using realistic parameters. ${ }^{24}$ The effect of the multilayer potential on the unhybridized free electrons is thus negligible.

By mimicking the band structure that is assumed in the simple models we retrieve a nearly vanishing ballistic MR. In the correct calculations the free electrons hybridize with the $d$ electrons, which results in a strong coupling to the "magnetic lattice", and a greatly enhanced reflection at the interfaces. GMR in the ballistic regime is thus induced by hybridization and theories that neglect this hybridization do not describe the effect correctly. The effect of the hybridization can be (partially) mimicked by the Kronig-Penney model only by adopting potential steps that are much larger than the real ones.

\section{D. (111) orientation}

It is straightforward to obtain results for different crystal orientations. In Fig. 16 the projections of the Fermi surfaces of a (111) oriented $\mathrm{Co}_{3} / \mathrm{Cu}_{3}$ multilayer are shown. The quali-

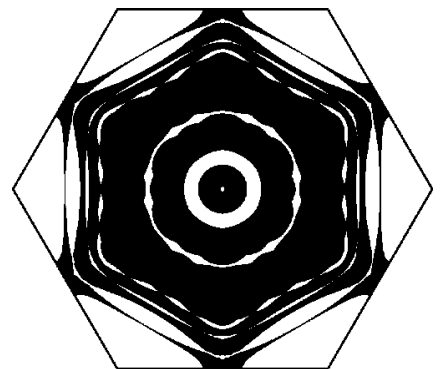

(a)

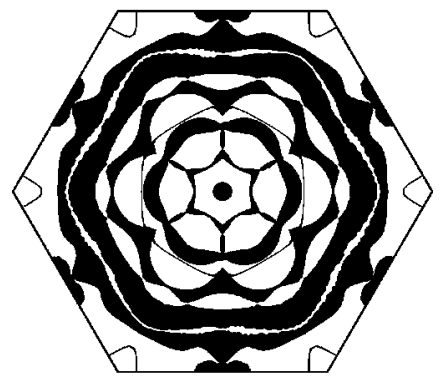

(b)

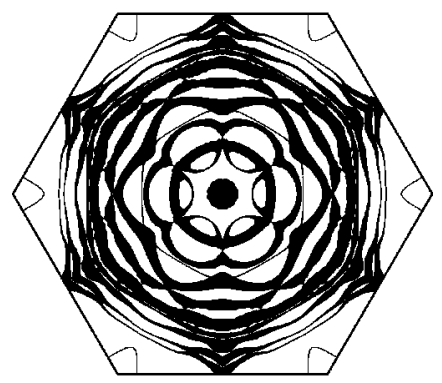

(c)

FIG. 16. Projections inside the first Brillouin zone of different Fermi surfaces for a (111) oriented $\mathrm{Co}_{3} / \mathrm{Cu}_{3}$ multilayer on a plane parallel to the interfaces. (a) Majority spin and (b) minority spin in the parallel configuration; (c) either spin in the antiparallel configuration.

tative features of the projections are similar to the ones for the (100) orientation shown in Fig. 10, except that in the (111) orientation the rotational symmetry along the $z$ axis is sixfold. In Figs. 16(b) and 16(c) two very clear examples of strongly confined quantum-well states are observed. The calculated conductance in the CPP geometry are $G_{\text {maj }}=0.41$, $G_{\text {min }}=0.32$ and $G_{\mathrm{AP}}=0.26$, all in units of $10^{15} \Omega^{-1} \mathrm{~m}^{-2}$. This results in a CPP MR of $40 \%$. The MR in the CIP geometry is only $4 \%$.

\section{Fe/Cr MULTILAYERS}

The GMR effect was originally discovered in multilayers consisting of $\mathrm{Fe}$ and $\mathrm{Cr} .{ }^{10,11} \mathrm{In}$ this section results are presented for both (100) and (110) oriented Fe/Cr multilayers.

\section{A. Technical aspects}

Bulk $\mathrm{Fe}$ and $\mathrm{Cr}$ both have a bcc structure and their lattice constants differ by less than a percent. In the multilayer calculations we therefore neglect any tetragonal distortions and assume a common bcc lattice for $\mathrm{Fe}$ and $\mathrm{Cr}$ with a lattice 


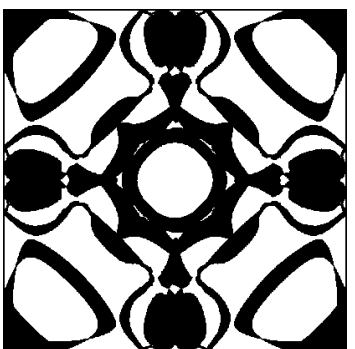

(a)

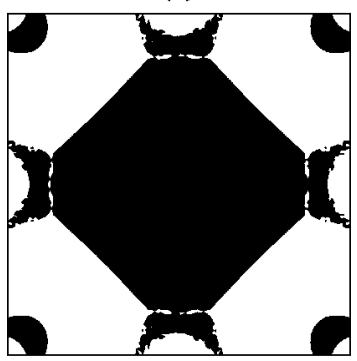

(b)

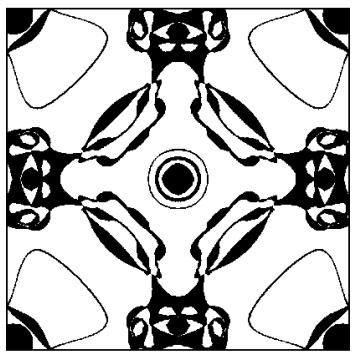

(c)

FIG. 17. Projections inside the first Brillouin zone of different Fermi surfaces for a (100) oriented $\mathrm{Fe}_{4} / \mathrm{Cr}_{4}$ multilayer on a plane parallel to the interfaces. (a) Majority spin and (b) minority spin in the parallel configuration; (c) either spin in the antiparallel configuration.

parameter of $2.844 \AA$, which is $99 \%$ of the average of the experimental bulk values, as was the case for $\mathrm{Co} / \mathrm{Cu}$ multilayers in Sec. V A.

In $\mathrm{Fe} / \mathrm{Cr}$ multilayers there is an extra complication due to the spin-density wave in $\mathrm{Cr}$, which causes an approximately two-monolayer oscillation of the magnetization along the (100) direction. The magnetic moment of a $\mathrm{Cr}$ monolayer at an interface is strongly antiferromagnetically coupled to the moment of the adjacent Fe layer. Since adjacent $\mathrm{Cr}$ monolayers couple antiferromagnetically, the spin-density wave is frustrated for an even number of $\mathrm{Cr}$ monolayers in the $\mathrm{P}$ configuration and for an odd number in the AP configuration. In the other magnetic configurations the spin-density wave fits nicely in between adjacent Fe layers. To take the spindensity wave into account correctly we perform the calculations in both the $\mathrm{P}$ and the AP configuration selfconsistently.

All calculations in this section are performed using an spd basis. The results we present for the CIP geometry are calculated along a (100) direction; we checked that the results along other CIP directions are not very different.

\section{B. (100) orientation}

In Fig. 17 the projections of the Fermi surfaces for a (100)
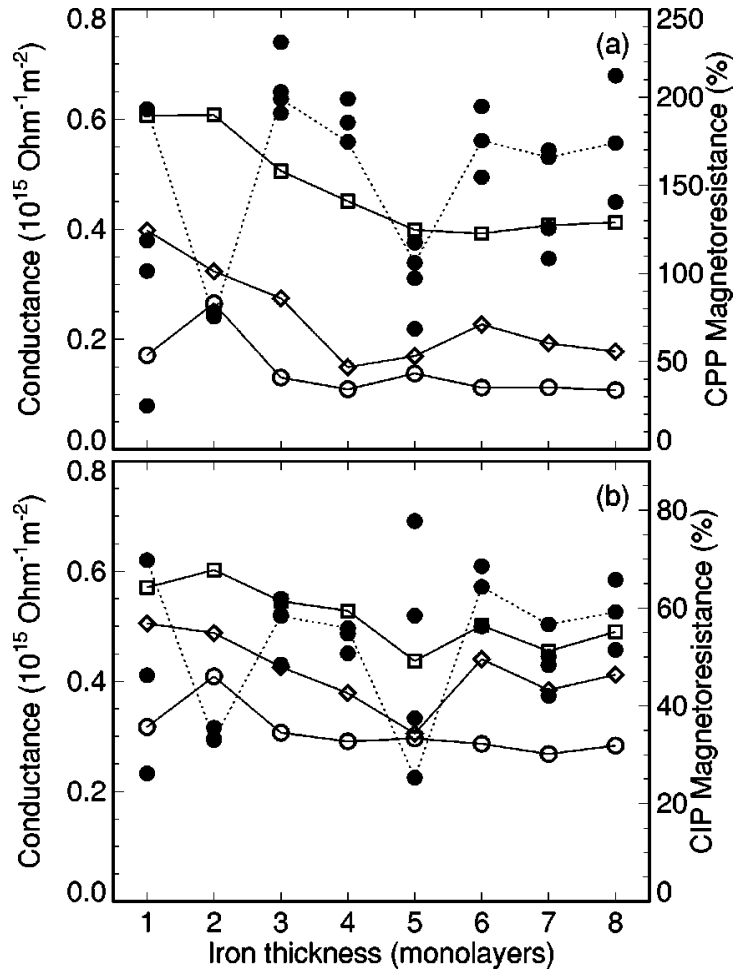

FIG. 18. The iron layer thickness dependence for (100) oriented $\mathrm{Fe}_{n} / \mathrm{Cr}_{6}$ multilayers of the MR (filled circles connected by dotted line) and of the conductances for the majority (diamonds) and the minority (squares) spin in the parallel configuration and for both spins in the antiparallel configuration (open circles). The filled circles that are not connected by the dotted line are the MR values for $\mathrm{Fe}_{n} / \mathrm{Cr}_{m}$ multilayers with $m \neq 6$. (a) CPP geometry; (b) CIP geometry.

oriented $\mathrm{Fe}_{4} / \mathrm{Cr}_{4}$ multilayer are shown. In contrast to the majority-spin projections for $\mathrm{Co} / \mathrm{Cu}$ multilayers it is impossible to analyze the projections for $\mathrm{Fe} / \mathrm{Cr}$ multilayers in terms of simple models because the Fermi surfaces of both Fe and $\mathrm{Cr}$ are very complicated for either spin direction. Figure 17 shows a nice example of quantum-well formation: the majority-spin states near the corners of the Brillouin zone that are propagating in the $\mathrm{P}$ configuration are confined to form quantum-well states in the AP configuration because of the absence of corresponding minority-spin states.

In Table II the conductances and MR values are summarized for a large number of (100) oriented $\mathrm{Fe}_{n} / \mathrm{Cr}_{m}$ multilayers. Most calculations are for $n+m=$ even because for $n+m=$ odd the unit cell in the $\mathrm{P}$ configuration contains $2(n+m)$ atoms compared to $n+m$ atoms for $n+m=$ even. Except for very thin layers the calculated MR in the CPP geometry lies between $70 \%$ and $230 \%$, again comparable to the experimental values in the diffusive regime. ${ }^{17}$ In contrast to $\mathrm{Co} / \mathrm{Cu}$ where most of the current is carried by the majority-spin electrons, the conductance in $\mathrm{Fe} / \mathrm{Cr}$ is dominated by the minority-spin bands, which can be understood from the better matching of the bulk band structures of Fe and $\mathrm{Cr}$ for this spin direction.

The large number of different layer thicknesses in Table II makes it possible to analyze the results as a function of $n$, $m$, and $n+m$. We find that the CPP MR depends mainly on the Fe thickness. Figure 18(a) shows the dependence of the 
TABLE II. Values of the calculated conductances $G$ (in units $10^{15} \Omega^{-1} \mathrm{~m}^{-2}$ ) in both the CPP and the CIP geometry for several (100) oriented $\mathrm{Fe}_{n} / \mathrm{Cr}_{m}$ multilayers. $G_{\text {maj }}\left(G_{\text {min }}\right)$ : conductance of the majority (minority) spin in the parallel configuration. $G_{\mathrm{AP}}$ : conductance per spin in the antiparallel configuration (both spins are the same $)$. The magnetoresistance is defined as $M R=\left(G_{\text {maj }}\right.$ $\left.+G_{\min }-2 G_{\mathrm{AP}}\right) / 2 G_{\mathrm{AP}}$.

\begin{tabular}{|c|c|c|c|c|c|c|c|c|}
\hline & & & P & & & & IP & \\
\hline & $G_{\text {maj }}$ & $G_{\text {min }}$ & $G_{\mathrm{AP}}$ & MR & $G_{\text {maj }}$ & $G_{\min }$ & $G_{\mathrm{AP}}$ & MR \\
\hline${ }_{1} / \mathrm{Cr}_{3}$ & 0.52 & 0.29 & 0.32 & $25 \%$ & 0.58 & 0.27 & 0.45 & $-6 \%$ \\
\hline $\mathrm{e}_{1} / \mathrm{Cr}_{5}$ & 0.40 & 0.44 & 0.21 & $101 \%$ & 0.48 & 0.44 & 0.36 & $26 \%$ \\
\hline $\mathrm{e}_{1} / \mathrm{Cr}_{6}$ & 0.41 & 0.62 & 0.17 & $193 \%$ & 0.51 & 0.58 & 0.32 & $70 \%$ \\
\hline $\mathrm{e}_{1} / \mathrm{Cr}_{7}$ & 0.32 & 0.49 & 0.18 & $119 \%$ & 0.41 & 0.51 & 0.32 & $46 \%$ \\
\hline $\mathrm{e}_{2} / \mathrm{Cr}_{4}$ & 0.48 & 0.61 & 0.31 & $77 \%$ & 0.58 & 0.60 & 0.44 & $36 \%$ \\
\hline $\mathrm{e}_{2} / \mathrm{Cr}_{6}$ & 0.33 & 0.62 & 0.27 & $75 \%$ & 0.50 & 0.61 & 0.42 & $33 \%$ \\
\hline $\mathrm{e}_{2} / \mathrm{Cr}_{8}$ & 0.28 & 0.62 & 0.25 & $78 \%$ & 0.41 & 0.63 & 0.39 & $33 \%$ \\
\hline $\mathrm{e}_{3} / \mathrm{Cr}_{3}$ & 0.36 & 0.49 & 0.13 & $231 \%$ & 0.45 & 0.49 & 0.29 & $62 \%$ \\
\hline $\mathrm{Fe}_{3} / \mathrm{Cr}_{5}$ & 0.26 & 0.53 & 0.14 & $191 \%$ & 0.36 & 0.57 & 0.31 & $48 \%$ \\
\hline $\mathrm{e}_{3} / \mathrm{Cr}_{6}$ & 0.28 & 0.52 & 0.13 & $199 \%$ & 0.43 & 0.56 & 0.31 & $58 \%$ \\
\hline $\mathrm{e}_{3} / \mathrm{Cr}_{7}$ & 0.18 & 0.60 & 0.13 & $203 \%$ & 0.34 & 0.65 & 0.31 & $59 \%$ \\
\hline $\mathrm{e}_{4} / \mathrm{Cr}_{4}$ & 0.26 & 0.41 & 0.12 & $185 \%$ & 0.48 & 0.47 & 0.31 & $55 \%$ \\
\hline $\mathrm{ee}_{4} / \mathrm{Cr}_{6}$ & 0.15 & 0.46 & 0.11 & $175 \%$ & 0.39 & 0.54 & 0.30 & $56 \%$ \\
\hline $\mathrm{e}_{4} / \mathrm{Cr}_{8}$ & 0.16 & 0.46 & 0.10 & $199 \%$ & 0.37 & 0.54 & 0.30 & $51 \%$ \\
\hline $\mathrm{Fe}_{5} / \mathrm{Cr}_{3}$ & 0.23 & 0.42 & 0.15 & $117 \%$ & 0.45 & 0.48 & 0.26 & $78 \%$ \\
\hline $\mathrm{Fe}_{5} / \mathrm{Cr}_{5}$ & 0.17 & 0.42 & 0.15 & $\%$ & 0.35 & 0.52 & 0.27 & $58 \%$ \\
\hline $\mathrm{e}_{5} / \mathrm{Cr}_{6}$ & 0.17 & 0.41 & 0.14 & $106 \%$ & 0.31 & 0.45 & 0.30 & $25 \%$ \\
\hline $\mathrm{Fe}_{5} / \mathrm{Cr}_{7}$ & 0.14 & 0.40 & 0.16 & $69 \%$ & 0.31 & 0.51 & 0.30 & $38 \%$ \\
\hline $\mathrm{Fe}_{6} / \mathrm{Cr}_{4}$ & 0.28 & 0.44 & 0.12 & $195 \%$ & 0.47 & 0.50 & 0.29 & $68 \%$ \\
\hline $\mathrm{e}_{6} / \mathrm{Cr}_{6}$ & 0.23 & 0.40 & 0.11 & $175 \%$ & 0.45 & 0.51 & 0.29 & $64 \%$ \\
\hline $\mathrm{e}_{6} / \mathrm{Cr}_{8}$ & 0.19 & 0.43 & 0.12 & $155 \%$ & 0.39 & 0.51 & 0.29 & $56 \%$ \\
\hline $\mathrm{Fe}_{7} / \mathrm{Cr}_{3}$ & 0.16 & 0.44 & 0.14 & $108 \%$ & 0.36 & 0.49 & 0.30 & $42 \%$ \\
\hline $\mathrm{Fe}_{7} / \mathrm{Cr}_{5}$ & 0.15 & 0.40 & 0.12 & $125 \%$ & 0.32 & 0.51 & 0.28 & $48 \%$ \\
\hline $\mathrm{Fe}_{7} / \mathrm{Cr}_{6}$ & 0.20 & 0.41 & 0.11 & $166 \%$ & 0.39 & 0.46 & 0.27 & $57 \%$ \\
\hline $\mathrm{Fe}_{7} / \mathrm{Cr}_{7}$ & 0.14 & 0.42 & 0.10 & $170 \%$ & 0.31 & 0.50 & 0.27 & $50 \%$ \\
\hline $\mathrm{Fe}_{8} / \mathrm{Cr}_{4}$ & 0.27 & 0.42 & 0.11 & $212 \%$ & 0.48 & 0.48 & 0.29 & $66 \%$ \\
\hline $\mathrm{Fe}_{8} / \mathrm{Cr}_{6}$ & 0.18 & 0.42 & 0.11 & $174 \%$ & 0.42 & 0.50 & 0.29 & $59 \%$ \\
\hline $\mathrm{Fe}_{8} / \mathrm{Cr}_{8}$ & 0.17 & 0.42 & 0.12 & $140 \%$ & 0.40 & 0.50 & 0.30 & $51 \%$ \\
\hline
\end{tabular}

conductances and the MR in the CPP geometry on $n$ for (100) oriented $\mathrm{Fe}_{n} / \mathrm{Cr}_{6}$ multilayers and also the CPP MR for $\mathrm{Fe}_{n} / \mathrm{Cr}_{m}$. The thickness dependence of the conductances resembles those for $\mathrm{Co} / \mathrm{Cu}$ multilayers in Fig. 11(a) and those for the Kronig-Penney model in Fig. 6(a). A pronounced quantum size effect is found in the CPP MR mainly due to oscillations in $G_{\text {maj }}$ and $G_{\mathrm{AP}}$. The amplitude of the oscillations in the MR for $n=2-6$ is larger than the spread in the MR due to variations in the $\mathrm{Cr}$ thickness. The relatively weak dependence of the MR on the Cr thickness implies that the spin-density wave being frustrated or not makes only a minor difference to the GMR effect. The fact that the oscillations can be measured for a constant $\mathrm{Cr}$ thickness is advantageous for experimental observation of the quantum-size effect in the MR because the Cr thickness can be chosen such that the interlayer exchange coupling is antiferromagnetic for all Fe thicknesses.

Table II also contains the results for the CIP geometry. All CIP conductances are smaller than the weighted averages of the bulk conductances for $\mathrm{Fe}$ and $\mathrm{Cr}$, as was the case for the minority spin in the $\mathrm{Co} / \mathrm{Cu}$ multilayers. We tried to fit the data in Table II by the simple model of effective bulk conductances in parallel, but the calculated layer thickness dependence does not follow such simple behavior, which probably indicates that our calculations are not in the asymptotic regime. Figure 18(b) shows the results for the CIP geometry as a function of the Fe thickness. The spread in MR as $m$ is changed (for constant $n$ ) compared to the amplitude of the oscillations in the MR as a function of $n$ is larger than for the CPP geometry. The most surprising result from Table II and Fig. 18(b) is that the calculated CIP MR lies in between 25\% and $80 \%$, in contrast to the nearly vanishing CIP MR for $\mathrm{Co} / \mathrm{Cu}$ multilayers. The only exception is the $\mathrm{Fe}_{1} / \mathrm{Cr}_{3}$ multilayer for which the CIP MR is even negative, an atypical result that can be ascribed to the very thin layers. The calculated values are substantially smaller than the maximum observed value of $220 \%$. $^{74}$

A CIP MR of around 50\% for large layer thicknesses can be interpreted in terms of the backscattering mechanism that was discussed in Sec. V in relation to Fig. 12. Whereas for $\mathrm{Co} / \mathrm{Cu}$ multilayers the intersheet scattering is restricted to the Co minority-spin electrons, for $\mathrm{Fe} / \mathrm{Cr}$ multilayers it occurs for either spin direction in both $\mathrm{Fe}$ and Cr. Particularly in the nonmagnetic $(\mathrm{Cu}$ or $\mathrm{Cr})$ layer the reflection is expected to be sensitive to the magnetic configuration because this layer is directly adjacent to two magnetic layers. In $\mathrm{Cr}$, which has multiple sheets of Fermi surface, reflection can give rise to a reduction of the CIP conductance, which makes an important difference between $\mathrm{Co} / \mathrm{Cu}$ and $\mathrm{Fe} / \mathrm{Cr}$ multilayers. However, such a reduction does not only require a finite reflection but this reflection should also couple different sheets of Fermi surface. We expect that the intersheet scattering is relatively strong for the (100) orientation because of the atomic structure of the interface as represented schematically in Fig. 19. In the (100) orientation of a bcc multilayer the density of atoms in the atomic planes is low whereas the different atomic planes are close together, which gives rise to an interface that is not very flat, even though it is atomically sharp. From a Kronig-Penney type of model it can be understood that interfaces that are modulated periodically with a period larger than $\lambda_{F} / 2$ give rise to scattering between different sheets of the back-folded Fermi surface. The amplitude of this intersheet scattering depends on the height of the potential step and on the amplitude of the interface modulation. The contribution of the "potential step" to the intersheet scattering depends on the magnetic configuration and the spin direction and can in combination with the large modulation amplitude in the (100) orientation give rise to a considerable decrease in the CIP conductance when switching from the $\mathrm{P}$ to the AP configuration. The large values we obtain for the CIP MR indicate that this mechanism is quite effective in (100) oriented $\mathrm{Fe} / \mathrm{Cr}$ multilayers. Note that the CIP MR that arises from the modulation of the interface has the same origin as the MR in the CPP geometry.

We briefly discuss the effect of $s p-d$ hybridization on the transport properties of $\mathrm{Fe} / \mathrm{Cr}$ multilayers. For a (100) oriented $\mathrm{Fe}_{4} / \mathrm{Cr}_{4}$ multilayer the MR in both the CPP and the CIP geometry is approximately $65 \%$ when the hybridization is switched off. These large values for the MR arise from the $d$ electrons that are present at $E_{F}$ for both spin directions in 

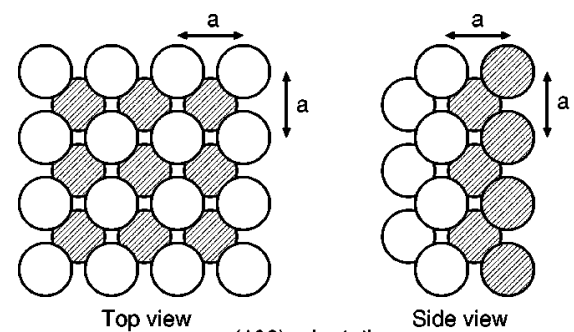

(100) orientation

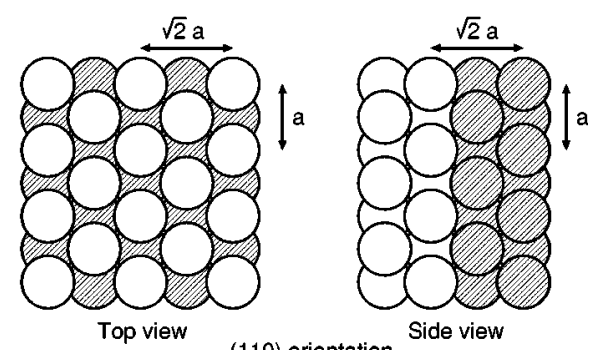

(110) orientation

FIG. 19. Schematic representation of the positions of the atoms near the interfaces of (100) and (110) oriented $\mathrm{Fe} / \mathrm{Cr}$ multilayers. The white and hatched spheres represent the $\mathrm{Fe}$ and $\mathrm{Cr}$ atoms, respectively. In the top views perpendicular to the interfaces one atomic plane on either side of the interface is shown. Twice as many atomic planes are shown in the side view.

both $\mathrm{Fe}$ and $\mathrm{Cr}$ and that contribute to the current in parallel to the free-electron-like $s p$ electrons. This is in contrast to the situation for $\mathrm{Co} / \mathrm{Cu}$ discussed in Sec. V C where the $d$ electrons are localized in the Co minority layers and do not contribute to the MR.

\section{C. (110) orientation}

To check our explanation for the large CIP MR for the (100) orientation, we performed calculations for the (110) orientation in which the density of atoms in the atomic planes is a factor $\sqrt{2}$ larger than in the (100) orientation while the different atomic planes are further apart by the same factor. The interfaces are therefore much flatter, as is clearly seen in Fig. 19. The calculated results for the (110) oriented $\mathrm{Fe}_{n} / \mathrm{Cr}_{n}$ multilayers are summarized in Table III. The conductances in the CIP geometry are larger than for the (100) orientation, especially for the majority-spin channel, which we ascribe to reduced intersheet scattering due to the smaller amplitude of the interface modulation. The multilayer conductance for the majority spin is slightly larger than the average of the bulk conductances of $\mathrm{Fe}$ and $\mathrm{Cr}$, which probably

TABLE III. Values of the calculated conductances $G$ (in units $10^{15} \Omega^{-1} \mathrm{~m}^{-2}$ ) in both the CPP and the CIP geometries for several (110) oriented $\mathrm{Fe}_{\mathrm{n}} / \mathrm{Cr}_{\mathrm{n}}$ multilayers. For explanation of the quantities, see Table II.

\begin{tabular}{lcccrcccc}
\hline \hline & \multicolumn{4}{c}{$\mathrm{CPP}$} \\
& $G_{\text {maj }}$ & $G_{\text {min }}$ & $G_{\mathrm{AP}}$ & \multicolumn{1}{c}{$\mathrm{MR}$} & $G_{\text {maj }}$ & $G_{\text {min }}$ & $G_{\mathrm{AP}}$ & $\mathrm{MR}$ \\
\hline $\mathrm{Fe}_{3} / \mathrm{Cr}_{3}$ & 0.34 & 0.45 & 0.18 & $120 \%$ & 0.61 & 0.56 & 0.53 & $9 \%$ \\
$\mathrm{Fe}_{4} / \mathrm{Cr}_{4}$ & 0.21 & 0.37 & 0.16 & $85 \%$ & 0.58 & 0.53 & 0.53 & $4 \%$ \\
$\mathrm{Fe}_{5} / \mathrm{Cr}_{5}$ & 0.24 & 0.36 & 0.15 & $94 \%$ & 0.60 & 0.52 & 0.53 & $6 \%$ \\
$\mathrm{Fe}_{6} / \mathrm{Cr}_{6}$ & 0.20 & 0.33 & 0.15 & $79 \%$ & 0.58 & 0.53 & 0.54 & $3 \%$ \\
\hline \hline
\end{tabular}

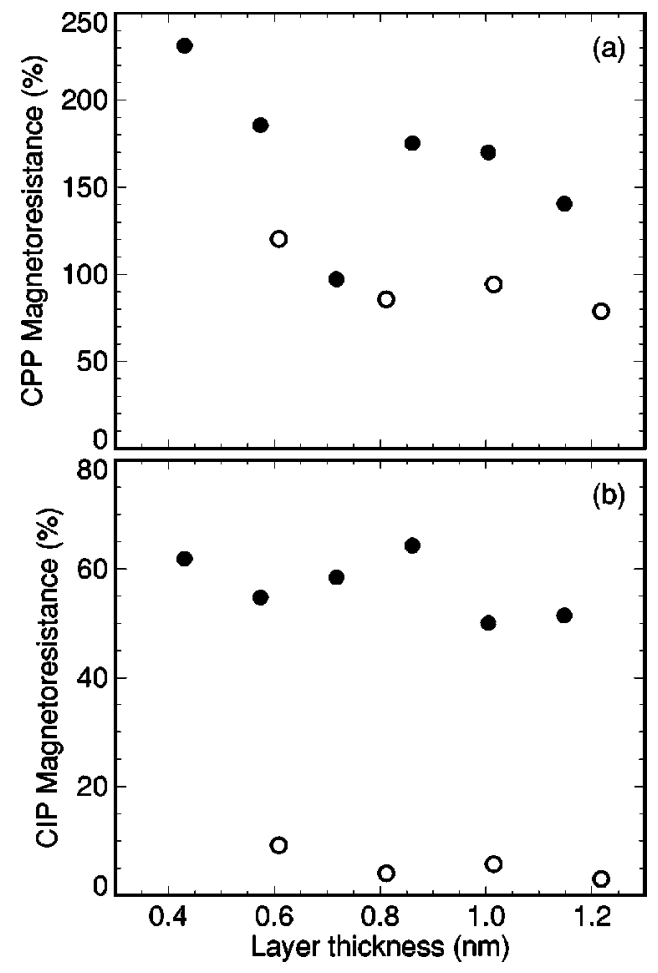

FIG. 20. The layer thickness dependences of (a) the CPP MR and (b) the CIP MR for $\mathrm{Fe}_{n} / \mathrm{Cr}_{n}$ multilayers in the (100) (filled circles) and (110) (open circles) orientation. Note that the layer thicknesses are plotted in nanometers because the thickness per monolayer is different for the two orientations.

indicates that our results are not in the asymptotic regime. In Fig. 20 the MR values for (100) and (110) oriented $\mathrm{Fe}_{n} / \mathrm{Cr}_{n}$ multilayers are compared. For the CIP geometry the difference between the two orientations is striking. The MR in the (110) orientation is only a few percent, as in the $\mathrm{Co} / \mathrm{Cu}$ multilayers, which we ascribe to the smaller sensitivity to the "potential steps" due to the flatter interfaces. The reduction of the multilayer conductance for the minority spin in the $\mathrm{P}$ configuration does not give rise to a significant MR, which probably indicates that this reduction mainly arises from states that are confined in the Fe minority layer. The difference between the (100) and the (110) orientations indicates that the CIP MR is very sensitive to the interface structure down to the monolayer level. For the CPP MR the difference is much less pronounced, which indicates that transport properties in this geometry are less sensitive to details of the interface structure.

Note that for fcc multilayers such as $\mathrm{Co} / \mathrm{Cu}$ the interface modulation is more similar for different crystal orientations than for bcc multilayers such as $\mathrm{Fe} / \mathrm{Cr}$. Due to the closepacked structure of the fcc lattice the interfaces for the (100) and (111) orientations are quite flat. The relation between intersheet scattering and the microscopic structure of the interface can be studied more explicitly by using methods that are capable of calculating transmission and reflection coefficients for single interfaces. ${ }^{75-77}$

\section{DISCUSSION}

We have shown that the conductance of classical ballistic point contacts can be calculated rigorously and parameter 
free by using electronic structure calculations in the localspin-density approximation. For $\mathrm{Co} / \mathrm{Cu}$ and $\mathrm{Fe} / \mathrm{Cr}$ multilayers in the CPP geometry a large ballistic GMR is predicted. We hope that this prediction will stimulate experimental studies of transport in the ballistic regime. Ballistic point contacts have already been fabricated for both nonmagnetic ${ }^{4}$ and magnetic ${ }^{5}$ bulk metals. The fabrication of magnetic multilayers of high structural quality in nanometer sized contacts remains an experimental challenge. ${ }^{13-15}$

Our calculations not only predict the values of the conductances, they also make a detailed analysis feasible of the role of the electronic structure in determining the transport properties. We find that the $s p-d$ hybridization is of crucial importance for the GMR in $\mathrm{Co} / \mathrm{Cu}$ multilayers. In $\mathrm{Fe} / \mathrm{Cr}$ the contribution of the $d$ electrons to the MR is important even in the unhybridized case. Neither of these effects is taken into account in theories that assume that the current is carried exclusively by free-electron-like $s$ electrons. For the CPP geometry the effect of hybridization can be (partially) mimicked by adopting potential steps which are much larger than the real ones. The ballistic CIP MR of (100) oriented Fe/Cr multilayers cannot be obtained by adjusting the parameters of the Kronig-Penney model because it is essential to take into account multiple bands. These results indicate that to describe the electrical transport in transition metal multilayers correctly the electronic structure should be taken into account in a realistic manner, preferably using $a b$ initio methods.

Although our calculations are directly applicable only to the ballistic regime we believe that they are also relevant for diffusive transport. It would be very surprising if none of the band-structure effects discussed above survives into the diffusive regime. For the CPP geometry, in particular, the influence of the band structure is very important. ${ }^{27,31} \mathrm{We}$ find that the ballistic MR saturates at about $90 \%$ for $\mathrm{Co} / \mathrm{Cu}$ multilayers and between $70 \%$ and $230 \%$ for $\mathrm{Fe} / \mathrm{Cr}$ multilayers, comparable with the experimental values for $\mathrm{Co} / \mathrm{Cu}$ (Refs. 71 and 72) and $\mathrm{Fe} / \mathrm{Cr}$ (Ref. 17) obtained in the diffusive regime at low temperatures. The CPP MR can thus be explained by differences in the number of conduction channels alone. Indeed, Asano, Oguri, and Maekawa ${ }^{31}$ have pointed out that the MR in the CPP geometry does not depend critically on interface defect scattering. Our results for (100) and (110) oriented $\mathrm{Fe} / \mathrm{Cr}$ multilayers also indicate that the CPP MR is not too sensitive to the interface structure. The results for the ballistic CPP MR should therefore be indicative for the experimental CPP MR. ${ }^{31}$ In terms of Eq. (8) this can be ascribed to a spin- and state-independent mean free number of traversed interfaces, which corresponds to spinindependent diffuse scattering that is located predominantly at the interfaces.

The calculated MR values in the CIP geometry are much smaller than the highest experimental values of $115 \%$ for $\mathrm{Co} / \mathrm{Cu}$ (Ref. 73) and 220\% for $\mathrm{Fe} / \mathrm{Cr}$ (Ref. 74) indicating that some additional scattering mechanism is important. Indeed, Asano, Oguri, and Maekawa ${ }^{31}$ find that the CIP MR depends critically on interface defect scattering. Our results for (100) and (110) oriented $\mathrm{Fe} / \mathrm{Cr}$ multilayers also indicate that the CIP MR is very sensitive to the interface morphology down to the monolayer level. In a sense, the "specular" $\vec{q}_{\|}$-conserving scattering between different sheets of Fermi surface for ideal interfaces resembles diffuse scattering at rough interfaces. Also experimentally the CIP MR strongly depends on the structural properties of the interface ${ }^{78}$ Still, we believe that the results derived in the ballistic limit have an even more general significance. Spin-dependent surface roughness scattering, which contributes to the GMR in the CIP geometry, is inextricably connected with the existence of strong spin-dependent reflection at ideal interfaces. ${ }^{79}$ This means that a large CPP effect as calculated by the present method is an important condition for a giant MR, either CPP or CIP.

In summary, we have shown that in the ballistic limit the transport properties can be evaluated rigorously using parameter-free calculations based on the local-spin-density approximation. The calculated CPP-MR is comparable to experimental values, even though defect scattering has been completely disregarded. We hope that this will stimulate experimental studies of transport in multilayers in this regime. To describe the effect it is of crucial importance to take into account the complete hybridization. We emphasize that no empirical parameters or other phenomenological input have been used.

\section{ACKNOWLEDGMENTS}

We thank Reinder Coehoorn and Martin Gijs for discussions. This work is part of the research program of the "Stichting voor Fundamenteel Onderzoek der Materie" (FOM), which is financially supported by the "Nederlandse Organisatie voor Wetenschappelijk Onderzoek" (NWO). We acknowledge benefits from the TMR Research Network on "Interface Magnetism', under Contract No. FMRX-CT960089 (DG12-MIHT).

\section{APPENDIX A: TETRAHEDRON METHOD}

In this appendix we describe an adaptation of the tetrahedron method ${ }^{59-61}$ used to calculate the Fermi surface projections. A uniform grid of $k$ points is adopted that divides the Brillouin zone into parallelepipeds. Each parallelepiped can be divided into six tetrahedra in four different ways over which we average the calculated projections afterwards. The contribution of each tetrahedron to the projection of the Fermi surface is calculated separately. For a particular energy band the energies at the four corners of the tetrahedron are denoted by $\varepsilon_{i}$, with $i$ the index of the corner. The $k$ point of the corner $i$ is denoted by $\vec{k}_{i}$. The labeling is such that $\varepsilon_{1} \leqslant \varepsilon_{2} \leqslant \varepsilon_{3} \leqslant \varepsilon_{4}$. We illustrate the calculation for the situation depicted schematically in Fig. 21 where the Fermi energy $E_{F}$ lies in between $\varepsilon_{3}$ and $\varepsilon_{4}$. The point $\vec{K}_{i}$ at which the Fermi surface intersects the line between $\vec{k}_{4}$ and $\vec{k}_{i}$ (with $i=1,2,3)$ is obtained by linear interpolation:

$$
\vec{K}_{i}=\vec{k}_{4}+\frac{\varepsilon_{4}-E_{F}}{\varepsilon_{4}-\varepsilon_{i}}\left(\vec{k}_{i}-\vec{k}_{4}\right) .
$$

From the vectors $\vec{K}_{i}$ the projection in the direction $\hat{n}$ of the Fermi surface inside the tetrahedron can be calculated as

$$
S(\hat{n})=\frac{1}{2}\left|\hat{n} \cdot\left(\vec{K}_{2}-\vec{K}_{1}\right) \times\left(\vec{K}_{3}-\vec{K}_{1}\right)\right| .
$$




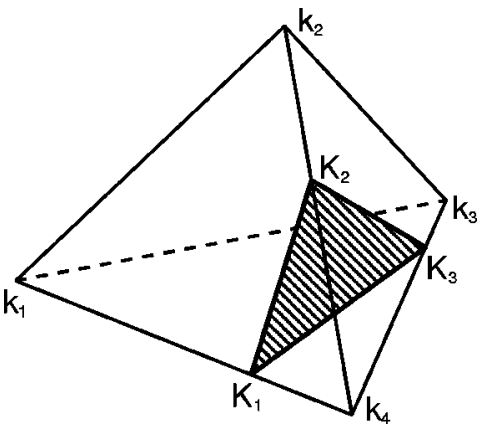

FIG. 21. Schematic representation of a tetrahedron used in the numerical calculation of the projection of the Fermi surface for the case that $\varepsilon_{3}<E_{F}<\varepsilon_{4}$. The points $\vec{k}_{i}$ represent the corners of the tetrahedron. The Fermi surface (hatched region) inside the tetrahedron is approximated by a triangle with vertices at the points $\vec{K}_{i}$.

The projections are calculated in a similar way when $E_{F}$ lies in between $\varepsilon_{2}$ and $\varepsilon_{3}$ or in between $\varepsilon_{1}$ and $\varepsilon_{2}$. The errors due to the linear interpolation cancel exactly for adjacent tetrahedra for the same sheet of Fermi surface, except when the tetrahedron contains an edge of the projected sheet. At a crossing of two sheets of Fermi surface the errors do not cancel either. For a uniform grid the total error vanishes for sufficiently fine meshes with the number of $k$ points to the power $-2 / 3$, which can be understood as the error that arises from approximating the projected area by the area of a polygon. In the main text we quote the values extrapolated to an infinite number of $k$ points unless stated otherwise.

To test the accuracy of the tetrahedron method we first calculate the Sharvin conductance in the (100) direction for a nearest-neighbor tight-binding model on a cubic lattice in three dimensions. The results are compared with an alternative calculation presented in Appendix B. The absolute difference between the two independent numerical calculations in Table IV is as small as $10^{-4}$. For the mesh containing $48^{3}$ $k$ points as was used in Fig. 3 the error is always smaller than $10^{-3}$ (see Table IV).

Figure 22 shows the dependence of the Sharvin conductance in different directions for bulk copper as a function of the number of $k$ points used in the Brillouin zone integration.

TABLE IV. The number of conducting channels per spin channel in the (100) direction for a nearest-neighbor tight-binding model on a cubic lattice in three dimensions as a function of the band filling. The values are calculated either using the tetrahedron method (for which both the results for a finite mesh of $48^{3} k$ points and the results extrapolated to an infinitely fine $k$ mesh are given) or by numerical evaluation of the integral in Eq. (B6). All values have an absolute accuracy of $10^{-4}$.

\begin{tabular}{cccc}
\hline \hline$\left|\varepsilon_{0}-E_{F}\right| / 2 t$ & $48^{3} k$ points & $\begin{array}{l}N\left(A / a^{2}\right) \\
\text { Extrapolated }\end{array}$ & Eq. (B6) \\
\hline 0.0 & 0.6311 & 0.6305 & 0.6304 \\
0.5 & 0.6071 & 0.6065 & 0.6066 \\
1.0 & 0.5000 & 0.5000 & 0.5000 \\
1.5 & 0.3081 & 0.3083 & 0.3083 \\
2.0 & 0.1845 & 0.1848 & 0.1848 \\
2.5 & 0.0848 & 0.0852 & 0.0852 \\
\hline \hline
\end{tabular}

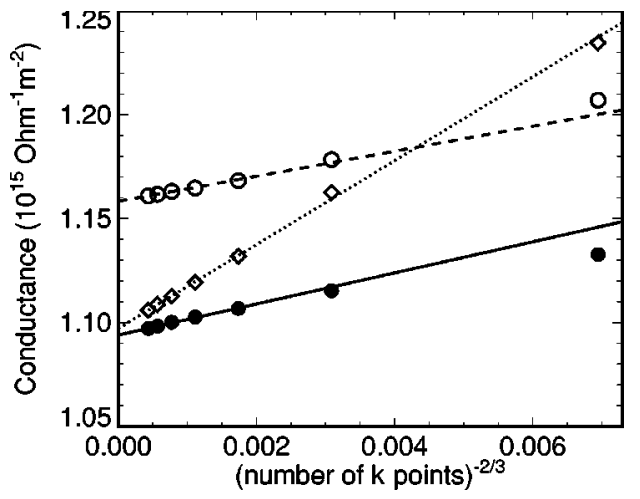

FIG. 22. The dependence of the calculated Sharvin conductance on the number of $k$ points used in the Brillouin zone integration. The solid, dashed, and dotted lines are linear fits to the calculated points for the (100) (closed circles), (110) (open circles), and (111) (diamonds) directions, respectively. The calculations were done using $12^{3}, 18^{3}, 24^{3}, 30^{3}, 36^{3}, 42^{3}$, and $48^{3} k$ points in the first Brillouin zone.

The difference between the extrapolated value and the value obtained using $48^{3} k$ points is smaller than $0.01 \times 10^{15}$ $\Omega^{-1} \mathrm{~m}^{-2}$. This provides an upper limit for the error arising from the Brillouin zone integration. Because of the systematic convergence for large numbers of $k$ points we expect the errors in the extrapolated values to be considerably smaller.

Plots similar to Fig. 22 were made for the results presented in the text. Good convergence was obtained also for the calculations for the multilayers. The error due to the Brillouin zone integration is typically smaller than $0.01 \times 10^{15} \Omega^{-1} \mathrm{~m}^{-2}$. Our adaptation of the tetrahedron method is thus sufficiently accurate for our present calculations.

\section{APPENDIX B: 3D TIGHT-BINDING MODEL}

In this appendix we derive a simple expression for the Sharvin conductance in the (100) direction for a nearestneighbor tight-binding model in three dimensions. This calculation is similar to the method used to simplify the expression for the density of states. ${ }^{80}$ The dispersion relation is

$$
\varepsilon_{\mathrm{TB}}(\vec{q})=\varepsilon_{0}-2 t\left(\cos q_{x} a+\cos q_{y} a+\cos q_{z} a\right),
$$

with $\varepsilon_{0}$ the on-site potential, $t$ the hopping matrix element, and $a$ the lattice constant. Since the projections of the constant energy surfaces are much more complicated than in the two-dimensional case, the ballistic conductance is now calculated directly from Eq. (4). For transport in the $x$ direction

$$
|\hat{n} \cdot \vec{v}(\vec{q})|=\frac{2 t a}{\hbar}\left|\sin q_{x} a\right| .
$$

The $\delta$ function is expressed as a Fourier integral:

$$
\delta(z)=\frac{1}{2 \pi} \int_{-\infty}^{\infty} d s e^{i z s} .
$$

Subsequently the integrations over the components of the Bloch vector $\vec{q}$ are carried out by using the standard integrals 


$$
\int_{0}^{\pi} d \phi e^{-i z \cos \phi} \sin \phi=2 \frac{\sin z}{z}
$$

and

$$
\int_{0}^{\pi} d \phi e^{-i z \cos \phi}=\pi J_{0}(z)
$$

where $J_{0}(z)$ is a Bessel function of order zero. The Sharvin conductance can then be expressed in terms of a single integration as

$$
G_{\mathrm{TB}}(100)=\frac{2 e^{2}}{h} \frac{A}{a^{2}} \frac{2}{\pi} \int_{0}^{\infty} d s \frac{\sin s}{s} J_{0}^{2}(s) \cos \frac{\left|\varepsilon_{0}-E_{F}\right| s}{2 t} .
$$

The numerical evaluation of the integral yields the values shown in Table IV. For $\left|\varepsilon-E_{F}\right|=2 t$ the Fermi surface is a slightly distorted octahedron of which the projection is exactly half the area of the first Brillouin zone. The value 0.5000 in Table IV is therefore exact.

We performed a similar calculation for the twodimensional situation. In that case also the final integral over the variable $s$ can be evaluated and Eq. (12a) is recovered.

\section{APPENDIX C: KRONIG-PENNEY MODEL FOR LARGE LAYER THICKNESSES}

In this appendix we calculate for the Kronig-Penney model the value of $G(\mathrm{CPP})$ in the limit of large layer thicknesses. The periodic potential is a periodic arrangement of scatterers, in this case potential barriers of height $U$ and width $d_{B}$ with interbarrier spacing $d_{A}$ (see Fig. 4). The transmission amplitude of one scatterer is written as $\sqrt{T\left(\vec{q}_{\|}\right)} \exp \left[i \delta\left(\vec{q}_{\|}\right)\right]$, with $T\left(\vec{q}_{\|}\right)$the transmission probability and $\delta\left(\vec{q}_{\|}\right)$a phase shift, which are functions of the mode index $\vec{q}_{\|}$. For a periodic arrangement of symmetric barriers the band structure is given by ${ }^{81}$

$$
\cos q_{\perp} d_{A}=\frac{1}{\sqrt{T\left(\vec{q}_{\|}\right)}} \cos \left[k_{\perp}^{A} d_{A}+\delta\left(\vec{q}_{\|}\right)\right]
$$

where $q_{\perp}$ is the $z$ component of the Bloch wave vector and $k_{\perp}^{A}$ is the perpendicular wave vector in the bulk of material $A$. The expectation value for the number of conducting channels $N$ at $\vec{q}_{\|}$is obtained by integrating over a small range of $\vec{q}_{\|}$and in three dimensions this corresponds for $d \gg \lambda_{F}$ to averaging over different layer thicknesses. By this procedure all quantum oscillations are integrated over and the asymptotic or average value is retained. The average (denoted by angular brackets) over $d_{A}$ can be obtained by simply counting the states with $\left|\cos q_{\perp} d_{A}\right| \leqslant 1$ in Eq. (C1), which yields

$$
\left\langle N\left(\vec{q}_{\|}\right)\right\rangle_{d_{A}}=1-\frac{2}{\pi} \arccos \sqrt{T\left(\vec{q}_{\|}\right)} .
$$

To perform the averaging over $d_{B}$ we note that the thickness dependent transmission probability for two equivalent scatterers in series (potential steps in this case) with a propagating state in between can be written as ${ }^{82}$

$$
T\left(\vec{q}_{\|}\right)=\left[1+b\left(\vec{q}_{\|}\right) \cos ^{2} \phi\left(\vec{q}_{\|}\right)\right]^{-1},
$$

where $\phi\left(\vec{q}_{\|}\right)$is half the phase accumulated between the potential steps for mode $\vec{q}_{\|}$, which depends linearly on $d_{B}$. The transmission probability averaged over $d_{B}$ is related to $b\left(\vec{q}_{\|}\right)$by

$$
\left\langle T\left(\vec{q}_{\|}\right)\right\rangle_{d_{B}}^{2}=\frac{1}{b\left(\vec{q}_{\|}\right)+1}
$$

as can be confirmed by averaging Eq. (C3) over $\phi\left(\vec{q}_{\|}\right)$. Averaging the number of conducting channels over $d_{B}$ yields

$$
\begin{aligned}
\left\langle N\left(\vec{q}_{\|}\right)\right\rangle_{d_{A}, d_{B}} & =\frac{1}{2 \pi} \int_{0}^{2 \pi} d \phi\left(\vec{q}_{\|}\right)\left\langle N\left(\vec{q}_{\|}\right)\right\rangle_{d_{A}} \\
& =1-\frac{4}{\pi^{2}} \int_{0}^{\pi / 2} d \varphi \arctan \left[\sqrt{b\left(\vec{q}_{\|}\right)} \cos \varphi\right] .
\end{aligned}
$$

The total conductance is obtained by summing over the transverse modes. The average probability for a propagating state $\vec{q}_{\|}$to be transmitted over the potential barrier is given by

$$
\left\langle T\left(\vec{q}_{\|}\right)\right\rangle_{d_{B}}=\frac{2 k_{\perp}^{A} k_{\perp}^{B}}{\left(k_{\perp}^{A}\right)^{2}+\left(k_{\perp}^{B}\right)^{2}},
$$

in which

$$
k_{\perp}^{B}=\sqrt{\left(1-U / E_{F}\right) k_{F}^{2}-q_{\|}^{2}} .
$$

For the evanescent states the transmission probabilities drop to zero exponentially with increasing thickness of the barrier and they do not contribute to the conductance for large layer thicknesses. By integrating over $\vec{q}_{\|}$and by using Eqs. (C4)(C7) we obtain

$$
\begin{aligned}
\frac{G_{\mathrm{KP}}(C P P)}{G_{\mathrm{FE}}}= & 1-\frac{U}{E_{F}}-\frac{4 U}{\pi^{2} E_{F}} \int_{0}^{\pi / 2} d \varphi \\
& \times \int_{1}^{E_{F} / U} d s \arctan \frac{\cos \varphi}{2 \sqrt{s(s-1)}},
\end{aligned}
$$

This function is plotted in Fig. 6(b). The first term on the right-hand side of Eq. (C8) corresponds to the bulk conductance of material $A$. The second gives the reduction due to the states that are evanescent in material $B$ and do not contribute to the conductance. ${ }^{24}$ The last term describes the reduction in the conductance from the states that are propagating in both materials but are partially reflected at each interface. 
*Present and permanent address: Philips Research Laboratories, Prof. Holstlaan 4, 5656 AA Eindhoven, The Netherlands.

${ }^{\dagger}$ Present and permanent address: Faculty of Applied Physics, University of Twente, P.O. Box 217, 5600 AE Enschede, The Netherlands.

${ }^{1}$ Yu. V. Sharvin, Zh. Éksp. Teor. Fiz. 48, 984 (1965) [ Sov. Phys. JETP 21, 655 (1965)].

${ }^{2}$ For a review, see V. S. Tsoi, J. Bass, and P. Wyder, Adv. Phys. 41, 365 (1992).

${ }^{3}$ J. Heil, M. Primke, K. U. Würz, and P. Wyder, Phys. Rev. Lett. 74, 146 (1995).

${ }^{4}$ I. K. Yanson, Zh. Éksp. Teor. Fiz. 66, 1035 (1974) [ Sov. Phys. JETP 39, 506 (1974)].

${ }^{5}$ B. I. Verkin, I. K. Yanson, I. O. Kulik, O. I. Shklyarevski, A. A. Lysykh, and Yu. G. Naydyuk, Solid State Commun. 30, 215 (1979).

${ }^{6}$ For a review, see A. G. M. Jansen, A. P. van Gelder, and P. Wyder, J. Phys. C. 13, 6073 (1980).

${ }^{7}$ K. S. Ralls and R. A. Buhrman, Phys. Rev. Lett. 60, 2434 (1988).

${ }^{8}$ P. A. M. Holweg, J. A. Kokkedee, J. Caro, A. H. Verbruggen, S. Radelaar, A. G. M. Jansen, and P. Wyder, Phys. Rev. Lett. 67, 2549 (1991).

${ }^{9}$ K. M. Schep, P. J. Kelly, and G. E. W. Bauer, Phys. Rev. Lett. 74, 586 (1995).

${ }^{10}$ M. N. Baibich, J. M. Broto, A. Fert, F. Nguyen Van Dau, F. Petroff, P. Etienne, G. Creuzet, A. Friederich, and J. Chazelas, Phys. Rev. Lett. 61, 2472 (1988).

${ }^{11}$ G. Binasch, P. Grünberg, F. Saurenbach, and W. Zinn, Phys. Rev. B 39, 4828 (1989).

${ }^{12}$ For a review, see P. M. Levy, Solid State Phys. 47, 367 (1994).

${ }^{13}$ R. Louie, S. Upadhyay, and R. A. Buhrman, Bull. Am. Phys. Soc. 41, 304 (1996).

${ }^{14}$ M. V. Tsoi, A. G. M. Jansen, and J. Bass, J. Appl. Phys. 81, 5530 (1997).

${ }^{15}$ K. Wellock, S. J. C. Theeuwen, N. N. Gribov, J. Caro, R. P. van Gorkom, S. Radelaar, J. Garfield, B. J. Hickey, M. J. Walker, R. Coehoorn, and F. D. Tichelaar (unpublished).

${ }^{16}$ W. P. Pratt, Jr., S.-F. Lee, J. M. Slaughter, R. Loloee, P. A. Schroeder, and J. Bass, Phys. Rev. Lett. 66, 3060 (1991).

${ }^{17}$ M. A. M. Gijs, S. K. J. Lenczowski, and J. B. Giesbers, Phys. Rev. Lett. 70, 3343 (1993).

${ }^{18}$ For a review, see M. A. M. Gijs and G. E. W. Bauer, Adv. Phys. 46, 285 (1997).

${ }^{19}$ T. Ono and T. Shinjo, J. Phys. Soc. Jpn. 64, 363 (1995).

${ }^{20}$ R. E. Camley and J. Barnaś, Phys. Rev. Lett. 63, 664 (1989).

${ }^{21}$ P. M. Levy, S. Zhang, and A. Fert, Phys. Rev. Lett. 65, 1643 (1990).

${ }^{22}$ J. Inoue, A. Oguri, and S. Maekawa, J. Phys. Soc. Jpn. 60, 376 (1991).

${ }^{23}$ S. Zhang and P. M. Levy, J. Appl. Phys. 69, 4786 (1991).

${ }^{24}$ G. E. W. Bauer, Phys. Rev. Lett. 69, 1676 (1992).

${ }^{25}$ R. Q. Hood and L. M. Falicov, Phys. Rev. B 46, 8287 (1992); R. Q. Hood, L. M. Falicov, and D. R. Penn, ibid. 49, 368 (1994).

${ }^{26}$ T. Valet and A. Fert, Phys. Rev. B 48, 7099 (1993).

${ }^{27}$ S. Zhang and P. M. Levy, in Magnetic Ultrathin Films, Multilayers and Surfaces/Interfaces and Characterization, edited by B. T. Jonker, S. A. Chambers, R. F. C. Farrow, C. Chappert, R. Clarke, W. J. M. de Jonge, T. Egami, P. Grünberg, K. M. Krishnan, E. E. Marinero, C. Rau, and S. Tsunashima, MRS Symposia Proceedings No. 313 (Materials Research Society, Pittsburgh, 1993), p. 53.
${ }^{28}$ P. B. Visscher, Phys. Rev. B 49, 3907 (1994).

${ }^{29}$ A. Brataas and G. E. W. Bauer, Phys. Rev. B 49, 14684 (1994).

${ }^{30}$ A. Vedyayev, N. Ryzhanova, B. Dieny, P. Dauguet, P. Gandit, and J. Chaussy, Phys. Rev. B 55, 3728 (1997).

${ }^{31}$ Y. Asano, A. Oguri, and S. Maekawa, Phys. Rev. B 48, 6192 (1993); (private communication).

${ }^{32}$ H. Itoh, J. Inoue, and S. Maekawa, Phys. Rev. B 51, 342 (1995).

${ }^{33}$ T. N. Todorov, E. Yu. Tsymbal, and D. G. Pettifor, Phys. Rev. B 54, R12 685 (1996).

${ }^{34}$ T. Oguchi, J. Magn. Magn. Mater. 126, 519 (1993).

${ }^{35}$ W. H. Butler, J. M. MacLaren, and X.-G. Zhang, in Magnetic Ultrathin Films, Multilayers and Surfaces/Interfaces and Characterization (Ref. 27), p. 59.

${ }^{36}$ R. K. Nesbet, J. Phys.: Condens. Matter 6, L449 (1994).

${ }^{37}$ P. Zahn, I. Mertig, M. Richter, and H. Eschrig, Phys. Rev. Lett. 75, 2996 (1995).

${ }^{38}$ W. H. Butler, X.-G. Zhang, D. M. C. Nicholson, T. C. Schulthess, and J. M. MacLaren, Phys. Rev. Lett. 76, 3216 (1996).

${ }^{39}$ E. Yu. Tsymbal and D. G. Pettifor, Phys. Rev. B 54, 15314 (1996).

${ }^{40}$ R. Gómez Abal, A. M. Llois, and M. Weissmann, Phys. Rev. B 53, R8844 (1996).

${ }^{41}$ C. J. Schinkel, R. Hartog, F. H. A. M. Hochstenbach, J. Phys. F 4, 1412 (1974).

${ }^{42}$ M. Richter, P. Zahn, M. Diviš, and I. Mertig, Phys. Rev. B 54, 11985 (1996).

${ }^{43}$ V. N. Antonov, A. Ya. Perlov, P. M. Oppeneer, A. N. Yaresko, and S. V. Halilov, Phys. Rev. Lett. 77, 5253 (1996).

${ }^{44}$ A. R. Mackintosh, Phys. Rev. Lett. 9, 90 (1962).

${ }^{45}$ A. J. Freeman, J. O. Dimmock, and R. E. Watson, Phys. Rev. Lett. 16, 94 (1966).

${ }^{46}$ M. Weissmann, A. M. Llois, R. Ramírez, and M. Kiwi, Phys. Rev. B 54, 15335 (1996).

${ }^{47}$ J. M. Gallego, D. Lederman, S. Kim, and I. V. Schuller, Phys. Rev. Lett. 74, 4515 (1995).

${ }^{48}$ J. Mathon, Phys. Rev. B 55, 960 (1997).

${ }^{49}$ G. E. W. Bauer, A. Brataas, K. M. Schep, and P. J. Kelly, J. Appl. Phys. 75, 6704 (1994).

${ }^{50}$ K. M. Schep, P. J. Kelly, and G. E. W. Bauer, J. Magn. Magn. Mater. 140-144, 503 (1995).

${ }^{51}$ K. M. Schep, P. J. Kelly, and G. E. W. Bauer, in Magnetic Ultrathin Films, Multilayers and Surfaces, edited by A. Fert, H. Fujimori, G. Güntherodt, B. Heinrich, W. F. Egelhoff, Jr., E. E. Marinero, and R. L. White, MRS Symposia Proceedings No. 384 (Materials Research Society, Pittsburgh, 1995), p. 305.

${ }^{52}$ K. M. Schep, P. J. Kelly, and G. E. W. Bauer, J. Magn. Magn. Mater. 156, 385 (1996)

${ }^{53}$ Note that classical point contacts (with diameters much larger than the electron wavelength) differ from quantum point contacts (with diameters of the order of the electron wavelength) in that quantum size effects are not important and the electronic structure in the contact is bulklike. An example of a quantum size effect is conductance quantization, see Ref. 57. The fact that the bulk electronic structure applies to classical point contacts is illustrated by the resemblance between our results in Fig. 3 and those of Todorov in Fig. 2 of Ref. 62 where the effect of a finite diameter on the conductance is evaluated explicitly.

${ }^{54}$ G. Wexler, Proc. Phys. Soc. London 89, 927 (1966).

${ }^{55}$ M. Knudsen, Kinetic Theory of Gases (Methuen \& Co., London, 1950), p. 7.

${ }^{56}$ S. Datta, Electronic Transport in Mesoscopic Systems (Cam- 
bridge University Press, Cambridge, 1995).

${ }^{57}$ B. J. van Wees, H. van Houten, C. W. J. Beenakker, J. G. Williamson, L. P. Kouwenhoven, D. van der Marel, and C. T. Foxon, Phys. Rev. Lett. 60, 848 (1988).

${ }^{58}$ For a review, see C. W. J. Beenakker and H. van Houten, Solid State Phys. 44, 1 (1991).

${ }^{59}$ O. Jepsen and O. K. Andersen, Solid State Commun. 9, 1763 (1971).

${ }^{60}$ G. Lehmann and M. Taut, Phys. Status Solidi B 54, 469 (1972).

${ }^{61}$ P. E. Blöchl, O. Jepsen, and O. K. Andersen, Phys. Rev. B 49, 16223 (1994).

${ }^{62}$ T. N. Todorov, Phys. Rev. B 54, 5801 (1996).

${ }^{63}$ C. Kittel, Introduction to Solid State Physics (Wiley, New York, 1986).

${ }^{64}$ J. Mathon, M. Villeret, and H. Itoh, Phys. Rev. B 52, R6983 (1995).

${ }^{65}$ O. Jepsen, D. Glötzel, and A. R. Mackintosh, Phys. Rev. B 23, 2684 (1981).

${ }^{66}$ T. Oguchi, A. J. Freeman, G. W. Crabtree, J. Magn. Magn. Mater. $63 \&$ 64, 645 (1987).

${ }^{67}$ O. K. Andersen, Phys. Rev. B 12, 3060 (1975).

${ }^{68}$ O. K. Andersen, O. Jepsen, and D. Glötzel, in Highlights in Condensed Matter Theory, edited by F. Bassani, F. Fumi, and M. P. Tosi (North-Holland, Amsterdam, 1985), p. 59.

${ }^{69}$ V. L. Moruzzi, J. F. Janak, and A. R. Williams, Calculated Electronic Properties of Metals (Pergamon, New York, 1978), p. 176.
${ }^{70}$ G. H. O. Daalderop, P. J. Kelly, and M. F. H. Schuurmans, Phys. Rev. B 42, 7270 (1990).

${ }^{71}$ W. P. Pratt, Jr., S.-F. Lee, P. Holody, Q. Yang, R. Loloee, J. Bass, and P. Schroeder, J. Magn. Magn. Mater. 126, 406 (1993).

${ }^{72}$ M. A. M. Gijs, J. B. Giesbers, M. T. Johnson, J. B. F. aan de Stegge, H. H. J. M. Janssen, S. K. J. Lenczowski, R. J. M. van de Veerdonk, and W. J. M. de Jonge, J. Appl. Phys. 75, 6709 (1994).

${ }^{73}$ S. S. P. Parkin, Z. G. Li, and D. J. Smith, Appl. Phys. Lett. 58, 2710 (1991).

${ }^{74}$ R. Schad, C. D. Potter, P. Beliën, G. Verbanck, V. V. Moshchalkov, and Y. Bruynseraede, Appl. Phys. Lett. 64, 3500 (1994).

${ }^{75}$ P. Bruno, Phys. Rev. B 52, 411 (1995).

${ }^{76}$ M. D. Stiles, J. Appl. Phys. 79, 5805 (1996); Phys. Rev. B 54, 14679 (1996).

${ }^{77}$ J. B. A. N. van Hoof, Ph.D. thesis, Nijmegen, 1997.

${ }^{78}$ J. M. Colino, I. K. Schuller, R. Schad, C. D. Potter, P. Beliën, G. Verbanck, V. V. Moshchalkov, and Y. Bruynseraede, Phys. Rev. B 53, 766 (1996).

${ }^{79}$ Complications can arise when the magnetic moments of the interface atoms are modified due to the roughness, see R. Coehoorn, J. Magn. Magn. Mater. 151, 341 (1995).

${ }^{80}$ J. Callaway, Quantum Theory of the Solid State (Academic Press, San Diego, 1991), p. 22.

${ }^{81}$ N. W. Ashcroft and N. D. Mermin, Solid State Physics (Saunders College, Philadelphia, 1976), p. 146 (Chap. 8, Problem 1).

${ }^{82}$ J. A. Melsen and C. W. J. Beenakker, Physica B 203, 219 (1994). 